\title{
Quelques Cestodes nouveaux d'Oiseaux d'Asie
}

\author{
par
}

\section{O. SCHMELZ}

Avec 43 figures et 7 tableaux dans le texte

SOMMAIRE

Introduction

Paronia bocki n. sp.

Davainea baeri n. sp.

Raillietina (Raillietina) fausti n. sp. . . . . . . . . . . 157

Raillietina (Raillietina) clairae n. sp. . . . . . . . . . . . 161

Raillietina (Paroniella) siamensis n. sp............ . 166

Raillietina (Paroniella) pinsonae n. sp. . . . . . . . . . . . 171

Biuterina fuhrmanni n. sp. . . . . . . . . . . . . . . . . 175

Hymenolepis linderi n. sp. . . . . . . . . . . . . . . . . . 181

Diorchis anomala n. sp. . . . . . . . . . . . . . . . . . 184

Cladotaenia feuta Meggitt 1933 . . . . . . . . . . 191

A propos de la synonymie de Cladotaenia cylindracea (Bloch 1782) 194

Quelques remarques au sujet de Cladotaenia fania Meggitt 1933195

Considérations sur le genre Paracladotaenia Yamaguti 1935 . . 198

Liste comparative des espèces du genre Cladotaenia des Accipitriformes . . . . . . . . . . . . . 196

Bibliographie . . . . . . . . . . . . . . . . . . . . . . 199 


\section{INTRODUCTION}

Cette étude a été entreprise sur le conseil et sous la direction de M. le professeur O. Funrmann, professeur de Zoologie et d'Anatomie comparée à l'Université de Neuchâtel.

Les matériaux proviennent de deux collections. L'une a été faite par le professeur Ernest C. FAUST, anciennement professeur à l'Université de Peiping, actuellement à l'Université de Tulane (U.S.A.). L'autre, réunie par le comte Nils Gyldenstolpe, provient du Musée de Stockholm et nous a été aimablement envoyée par le professeur Sixten Bock, directeur de ce musée.

Disposant de matériaux très nombreux et variés, nous y avons trouvé neuf espèces nouvelles dans les genres Paronia, Davainea, Raillietina (Raillietina), Raillietina (Paroniella), Biuterina, Hymenolepis et Diorchis. Pour terminer notre travail, nous avons consacré un chapitre au genre Cladotaenia en complétant l'étude de Cladotaenia feuta Meggitt, et en émettant quelques remarques et considérations sur la synonymie de Cladotaenia cylindracea, sur l'étude de Cladotaenia fania Meggitt, et sur le genre Paracladotaenia Yamaguti.

Pour tous les genres de Cestodes auxquels nous avons eu affaire dans ce travail et chez lesquels il existait déjà plus de deux espèces, nous avons établi des listes comparatives aussi détaillées que possible dans le but de faciliter ainsi le travail des spécialistes qui se trouveront en présence de Cestodes appartenant à l'un ou l'autre de ces genres, et avec l'espoir que ce modeste travail sera de quelque utilité plus tard.

Le professeur Shen Tseng a eu entre les mains des matériaux de la même collection de Chine et y a puisé le sujet de plusieurs travaux que notre étude vient, en quelque sorte, compléter.

Les matériaux de Chine, probablement recueillis à l'état mort et conservés dans l'alcool pendant très longtemps, étaient légèrement macérés, tandis que les Cestodes du Siam, pris à l'état vivant 
et conservés dans le sublimé, étaient fortement contractés, mais en parfait état de conservation.

Que M. le professeur O. Funrmann me permette de lui témoigner, ici, ma profonde gratitude pour le dévouement avec lequel il n'a cessé de diriger et encourager mes efforts et qu'il veuille bien accepter l'hommage de mon respectueux attachement. Je remercie aussi très sincèrement M. le professeur J. G. BAER pour l'intérêt qu'il a bien voulu porter à mon travail, pour ses conseils éclairés et ses corrections. 
Paronia bocki n. sp.

Hôtes: $\left.\begin{array}{rl}\text { Megalaema virens (Bodd.) } \\ \text { Cyanops ramsayi (Wald.) }\end{array}\right\}$ Capitoniformes.

Localité: Siam.

La longueur totale est d'environ 70 à $75 \mathrm{~mm}$. Derrière le scolex il y a une zone non segmentée d'environ $1 \mathrm{~mm}$. Les premiers segments sont les moins larges; le premier mesure $0,7 \mathrm{~mm}$. Puis les segments s'élargissent, atteignent 3 à $5 \mathrm{~mm}$. et se rétrécissent de nouveau dans le dernier tiers du strobila. Finalement, le dernier proglottis n'a plus que $1,2 \mathrm{~mm}$. de large, mais, par contre, il est sensiblement plus allongé et sa longueur atteint $1 \mathrm{~mm}$. Dans les anneaux larges de 1,5 à $2 \mathrm{~mm}$. et longs de $230 \mu$ à $280 \mu$ on trouve les ébauches des organes sexuels. Les anneaux adultes mesurent 3 à $4 \mathrm{~mm}$. de large et sont longs de $280 \mu$ à $335 \mu$. Puis les organes femelles disparaissent progressivement et dans les segments de 4 à $5 \mathrm{~mm}$. de large et de $500 \mu$ à $580 \mu$ de long les deux utérus sont confondus, mais les œufs ne sont pas encore mûrs.

Le scolex qui ressemble sensiblement à celui de Paronia pycnonoti Yamaguti, 1935 dans ses contours, a un diamètre maximum de $800 \mu$. Les quatre ventouses sont ovalaires et ont un diamètre de $400 \mu \times 290 \mu$.

$\mathrm{Sys}$ tè m e m u s c u la i r e La musculature en général n'a rien de particulier si ce n'est que la musculature dorso-ventrale est très développée et semble l'être encore davantage latéralement,

FIG. 1-6.

Paronia bocki n. sp.: 1. coupe horizontale d'un segment jeune; 2. coupe horizontale d'un segment adulte; 3 . portion d'une coupe transversale passant par l'ovaire et la glande vitellogène; 4. portion latéral d'une coupe transversale, passant par la poche du cirre et le canal déférent; 5. coupe horizontale de la région latérale du segment, montrant les muscles dorso-ventraux; 6. coupe horizontale d'un segment gravide montrant l'utérus.

$\mathrm{C}=$ canal déférent; $\mathrm{ca}=$ corpuscule calcaire; $\operatorname{cov}=$ capsule ovarienne; $\mathrm{cp}=$ cellule $\mathrm{du}$ parenchyme; $\mathrm{dv}=$ muscles dorso-ventraux $;$ ov $=$ ovaire; $\mathrm{t}=$ testicules; $\mathrm{ut}=$ utérus; $\mathrm{v}=$ vagin. 
QUELQUES CESTODES NOUVEAUX D'OISEAUX D'ASIE
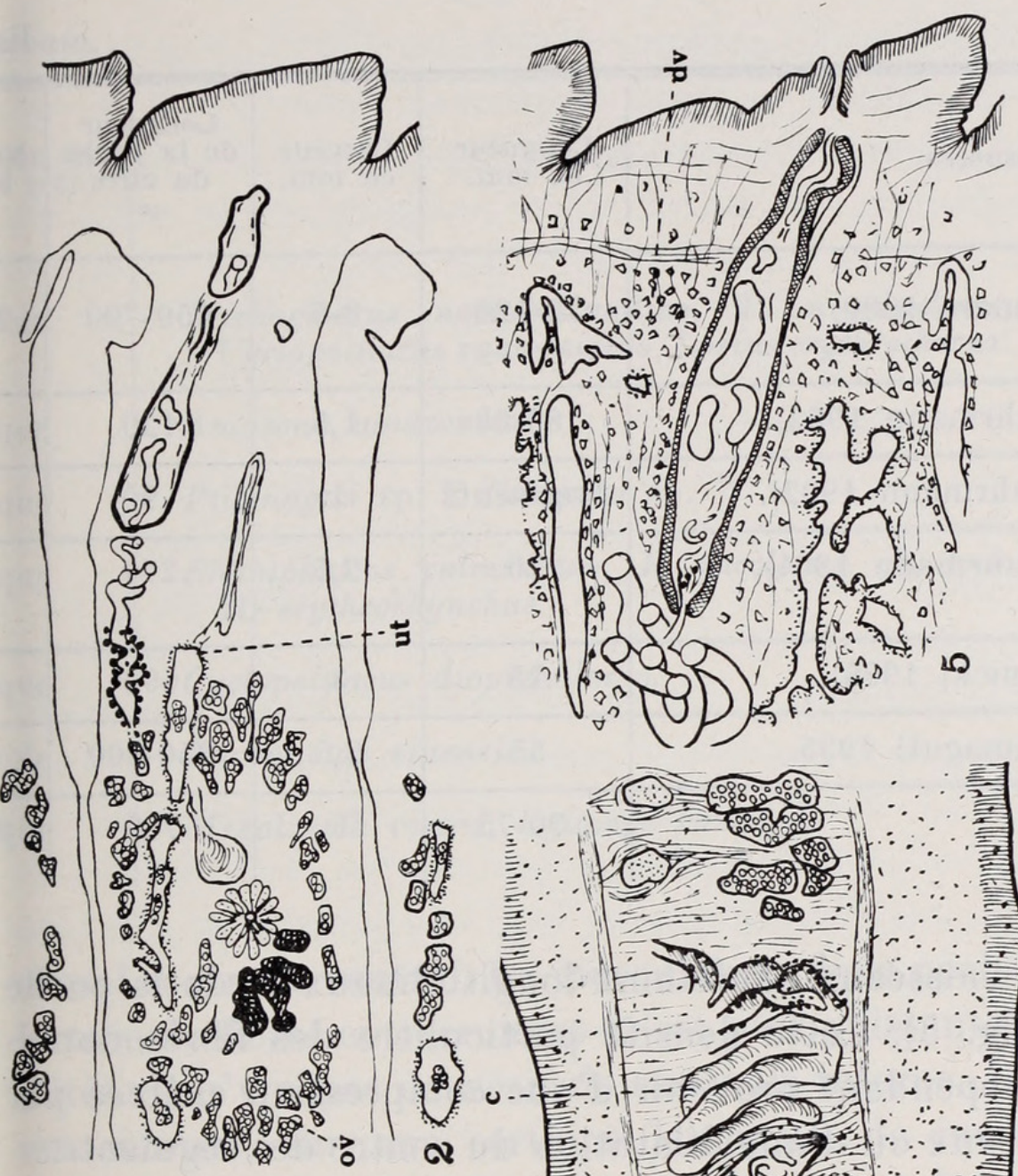
3.
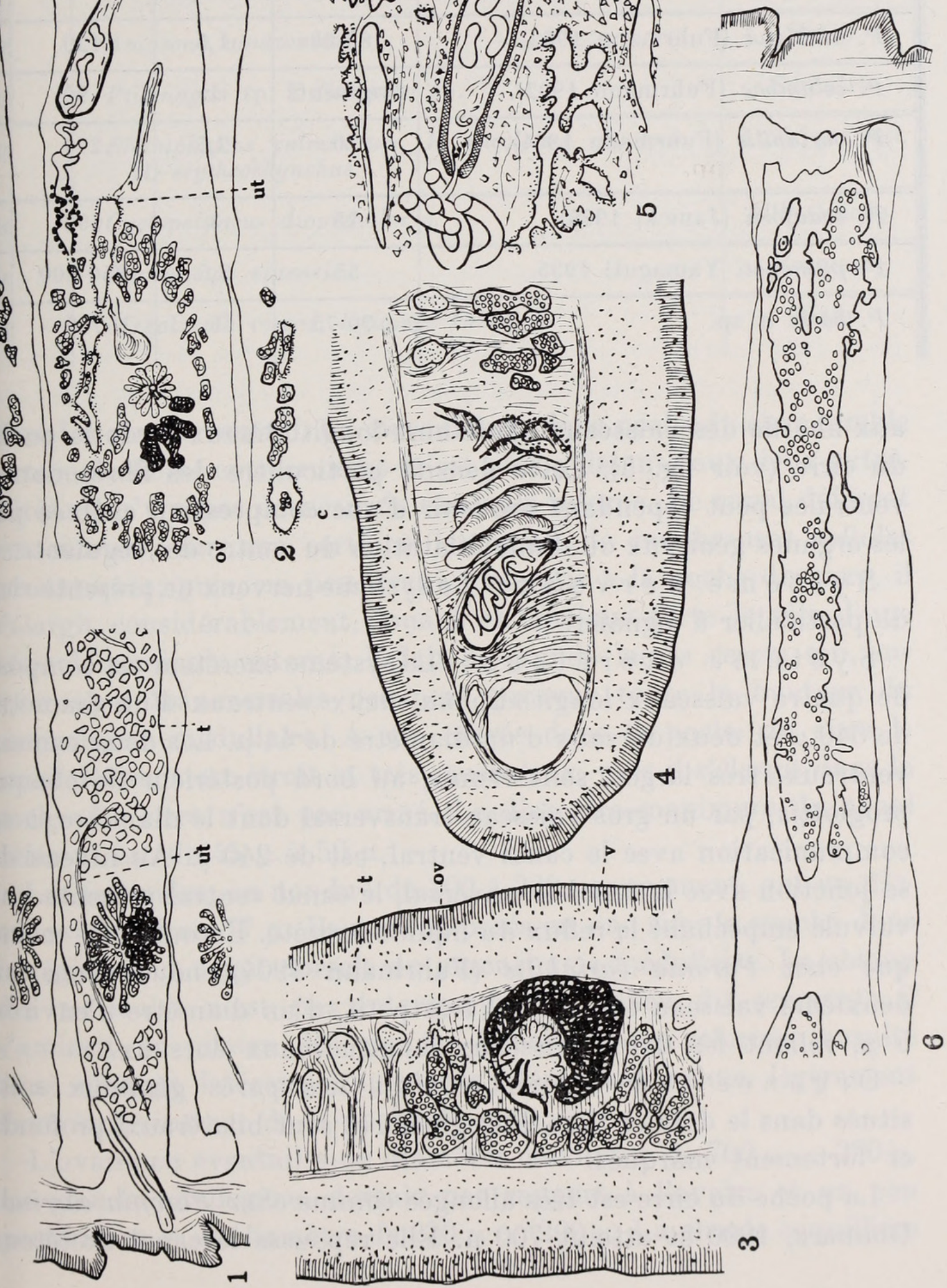


\begin{tabular}{|c|c|c|c|c|}
\hline Espèces & $\begin{array}{l}\text { Longueur } \\
\text { en mm. }\end{array}$ & $\begin{array}{l}\text { Largeur } \\
\text { en mm. }\end{array}$ & $\begin{array}{c}\text { Longueur } \\
\text { de la poche } \\
\text { du cirre } \\
\mu\end{array}$ & $\begin{array}{c}\text { Nor } \\
\text { tes }\end{array}$ \\
\hline P. carrinoi Diamare 1900. & $70-120$ & $3-5$ & $450-700$ & 14 \\
\hline P. ambigua (Fuhrmann 1902). & $80-60$ & 1,5 & 120 & \\
\hline P. columbae (Fuhrmann 1902). & fragments & 1 & 90 & \\
\hline P. variabilis (Fuhrmann 1904). & 70 & 2,5 & 270 & \\
\hline P. beauforti (Janicki 1906). & 18 & 3 & 600 & \\
\hline P. pycnonoti Yamaguti 1935. & 55 & 2,5 & $250-300$ & \\
\hline P. bocki n. sp. & $70-75$ & 5 & 700 & 20 \\
\hline
\end{tabular}

aux abords des vaisseaux excréteurs longitudinaux et de la poche du cirre (voir fig. 5). Cette densité particulière des fibres dorsoventrales peut cependant provenir d'une compression exercée par les organes génitaux ou d'une dilatation du centre des segments.

$\mathrm{S}$ y s t è m e $\mathrm{n}$ e $\mathrm{r} v$ e u $\mathrm{x}$ : Le système nerveux ne présente rien de particulier à signaler.

S y s t è m e e $\mathrm{x}$ c r é t e u r : Le système excréteur est composé de quatre vaisseaux longitudinaux, deux ventraux d'un diamètre de $340 \mu$ et deux dorsaux d'un diamètre de $44 \mu$. Les deux canaux ventraux, très larges, sont réunis, au bord postérieur de chaque proglottis, par un gros vaisseau transversal dont le diamètre, à sa communication avec le canal ventral, est de $240 \mu$. Au niveau de sa jonction avec le canal transversal, le canal ventral présente une valvule empêchant le reflux du liquide excrété. En outre, de même que chez Paronia variabilis (Fuhrmann 1904), nous avons un deuxième vaisseau transversal très petit, d'un diamètre d'environ $5 \mu$, reliant les deux vaisseaux longitudinaux dorsaux.

Organes reproducteurs: Les pores génitaux sont situés dans le dernier tiers du segment et sont bilatéraux, profonds et fortement marqués.

La poche du cirre est très allongée comme chez Paronia carrinoi Diamare, 1900 et atteint $700 \mu$. Elle repousse le canal excréteur 
aronia.

\begin{tabular}{|c|c|c|}
\hline $\begin{array}{l}\text { tion de } \\
\text { s mûr }\end{array}$ & Hôtes & Distribution \\
\hline ble & $\begin{array}{l}\text { Trichoglossus novaehollandiae, T. nigrigularis, } \\
\text { Cyclopsittacus suavissimus, Lorius erythrothorax. }\end{array}$ & $\begin{array}{l}\text { Australie et } \\
\text { Nlle_Guinée. }\end{array}$ \\
\hline que & Amazona amazonica. & Brésil \\
\hline que & Ptilonopus sp. ? Columba sp. ? & Sumatra \\
\hline que & $\begin{array}{l}\text { Rhamphastos culminatus, } R \text {. dicolorus, } R \text {. toco, } \\
\quad \text { R. erythrorhynchus. }\end{array}$ & Amérique sud. \\
\hline que & Cyclopsittacus diophthalmus. & Nlle_Guinée. \\
\hline ible & Pycnonotus sinensis. & Formose \\
\hline p & Megalaema sirens, Cyanops ramsayi. & Siam \\
\hline
\end{tabular}

dorsal contre le ventral. La musculature de la poche du cirre semble formée de fibres entrecroisées et est plus puissante aux deux extrémités. Il n'y a pas de vésicule séminale interne. Le canal déférent est entouré, sur une certaine longueur, de nombreuses cellules prostatiques, puis, un peu avant d'aboutir à la poche du cirre, il s'élargit considérablement, jouant en quelque sorte le rôle d'une vésicule séminale externe. Il fait de grands lacets serrés qui, sur les coupes transversales occupent presque toute la hauteur du parenchyme médullaire. A son entrée dans la poche du cirre le canal déférent est étroit et très musculeux, puis il s'élargit dans la poche. Le cirre n'est pas armé. Le diamètre maximum du canal déférent est de $45 \mu$ à $50 \mu$.

Les testicules, au nombre de 200 à 220 par segment, ont un diamètre d'environ $77 \mu$. Ils sont disposés en une simple couche dans la région tout à fait dorsale du parenchyme médullaire. Le champ des testicules n'est pas interrompu au milieu du segment; il s'amincit au niveau des glandes femelles pour reformer ensuite deux champs latéraux importants, dépassant même légèrement l'extrémité interne de la poche du cirre.

L'ovaire en éventail a un diamètre maximum de $700 \mu \times 290 \mu$. La glande vitellogène, dorsale par rapport à l'ovaire et un peu postérieure, a un diamètre de $220 \mu \times 200 \mu$. La glande coquillière 
mesure $120 \mu \times 90 \mu$. Le vagin, presque en ligne droite, passe entre la poche du cirre et le canal excréteur dorsal qui est refoulé contre le canal ventral. A la hauteur de la poche du cirre, le vagin forme deux renflements successifs bien marqués et qui semblent représenter des espèces de réservoirs, des réceptacles séminaux accessoires. Au voisinage de la glande coquillière, le vagin se dilate encore une fois en un réceptacle séminal long de $265 \mu$ à $275 \mu$ et d'un diamètre d'environ $100 \mu$.

L'utérus jeune est très étroit et allongé et descend obliquement vers le bord postérieur du segment, parallèlement à l'inclinaison de la poche du cirre (voir fig. 1). D'abord double, il forme peu à peu, au fur et à mesure qu'il se remplit d'œufs, des diverticules nombreux qui se ramifient et finissent par se rencontrer et fusionner au milieu du segment. Dans les proglottis mûrs l'utérus, devenu unique, remplit tout le parenchyme interne. Dans les derniers segments qui sont plus étroits et plus allongés, on trouve des œufs mûrs d'un diamètre d'environ $40 \mu$, renfermant un embryon de $20 \mu$ de diamètre. Il n'y a pas d'appareil piriforme.

D is cussion: Paronia bocki n. sp. ne peut être confondu avec $P$. carrinoi Diamare et $P$. pycnonoti Yamaguti dont l'utérus reste double dans les segments gravides. Il diffère de $P$. ambigua (Fuhrmann) et $P$. variabilis (Fuhrmann) par la longueur de la poche du cirre en même temps que par le nombre des testicules. S'il se rapproche de $P$. beauforti (Janicki) par la longueur de la poche du cirre, il s'en éloigne considérablement par la longueur totale et par le nombre des testicules. Il diffère aussi de $P$. columbae (Fuhrmann) par la longueur de la poche du cirre et par la largeur maxima du strobila. En outre, Paronia bocki n. sp. diffère de toutes les autres espèces du genre par la forme de l'utérus et la disposition des testicules. L'espace entre le bord latéral du segment et l'ovaire est assez vaste et rempli de testicules; l'utérus jeune, au lieu de la forme typique en fer à cheval contournant et embrassant intimement l'ovaire, forme une courbe très ouverte plus fortement inclinée dans la partie médiane du proglottis (voir fig. 1). Le champ des testicules est unique et ne s'interrompt pas au niveau de l'ovaire ni dans la ligne médiane comme c'est le cas chez certaines espèces du genre. Il s'y amincit seulement pour reformer ensuite deux grosses masses testiculaires latérales. L'utérus, double à son origine, 
devient unique. Les diverticules que forme cet utérus au cours de son développement se compliquent de nombreuses ramifications présentant une certaine symétrie avant de se rencontrer et de fusionner (voir fig. 6).

\section{Davainea baeri n. sp.}

Hôte: Gecinus nigrigenis (Wardl. Rams.) (Piciformes).

Localité: Siam.

Davainea baeri n. sp. est long de 3 à $3,6 \mathrm{~mm}$. et composé de 27 à 37 segments, ce qui représente le plus grand nombre de segments trouvé jusqu'à maintenant chez une espèce de ce genre. Le cou qui est très court et large mesure environ $100 \mu$ de long sur $300 \mu$ de large. Le premier segment a $15 \mu$ de long et $280 \mu$ de large, tandis que le dernier mesure de $315 \mu$ à $382 \mu$ de large et $282 \mu$ à $300 \mu$ de long selon l'individu. Les segments les plus larges ont $465 \mu$ à $647 \mu$.

Le scolex a un diamètre d'environ $300 \mu$. Le rostre mesure transversalement $232 \mu$ et a, lorsqu'il est évaginé, une longueur d'environ $95 \mu$. Il porte une double couronne d'environ 400 crochets de $10 \mu$ de long. Sur les coupes horizontales (fig. 8) on voit clairement que chacun de ces crochets est en relation avec une longue fibre musculaire permettant aux crochets certains mouvements. Le rostre présente, en outre, certaines particularités: Sa partie musculaire, comme c'est aussi le cas chez D. paucisegmentata Fuhrmann, 1909 et $D$. andrei Fuhrmann, 1933 n'a pas une forme allongée et plus ou moins cônique, mais la forme d'un épais disque. D'autre part, il est couvert de petites épines sur tout son pourtour (fig. 7). Les ventouses, relativement petites comme c'est le cas chez toutes les espèces de Davainea, sont situées au centre de quatre grosses protubérances saillant du scolex, et ont un diamètre de $90 \mu$ à $100 \mu$. Elles sont armées de 10 à 12 rangs de petits crochets mesurant $6 \mu$ à $7 \mu$ de long.

Systèm e n e rve u x : Sur nos coupes on ne voit que les deux troncs principaux qui sont très volumineux (fig. 9 et 10) ayant un diamètre de $20 \mu$ à $25 \mu$. 
S y s tèm e ex c r ét e u r: Le système excréteur est formé de deux vaisseaux dorsaux d'un diamètre de $3 \mu$ et de deux vaisseaux ventraux d'un diamètre de $12 \mu$. Ces derniers sont reliés par un canal transversal dans chaque proglottis. Un fait rarement observé vient compliquer de façon intéressante ce système. En effet, les coupes horizontales (fig. 8) montrent nettement que le système excréteur se prolonge jusque dans le rostre où il forme un anneau logé dans le parenchyme, mais enserrant très étroitement le disque musculaire du rostre. Il ne nous a pas été possible de découvrir la relation entre cet anneau et les autres canaux excréteurs, mais un certain nombre de diverticules extrêmement courts, observés sur le parcours de l'anneau, nous font supposer que l'appareil excréteur du rostre est relié aux autres vaisseaux par quelques canalicules très étroits. Les fibres musculaires des crochets descendent presque jusqu'au niveau de cet anneau excréteur.

S y s t èm e musculair e : La musculature n'a rien de particulier. La musculature longitudinale est formée de deux couches dont la délimitation est assez peu distincte. La couche externe montre des faisceaux contenant un nombre assez restreint de fibres, tandis que ceux de la couche interne apparaissent plus denses. La musculature dorsoventrale est assez faible et ne présente rien de particulier.

Organ es reprod u c t e u r s : Les premières ébauches des organes sexuels apparaissent immédiatement derrière le cou. Le développement des organes mâles est assez rapide. Dans le neuvième segment on voit nettement se détacher l'ébauche de la poche du cirre et des testicules représentés par de petits groupes de cellules se colorant plus fortement. Dans le onzième segment on voit apparaître l'ovaire.

L'atrium génital est unilatéral. Cette disposition est très rare dans ce genre puisqu'il n'a été constaté jusqu'à présent qu'un€ seule fois chez D. paucisegmentata Fuhrmann. Il débouche dans le quart antérieur du proglottis.

FIG. 7-11.

Davainea baeri n. sp.: 7. scolex; 8. coupe horizontale du scolex passant pas le rostre et montrant l'anneau excréteur dans le rostre; 9-10. coupe: transversales passant par les organes génitaux; 11. coupe horizontale : travers deux segments adultes. 
QUElQues CESTODES NOUVEAUX D'OISEAUX D'ASIE

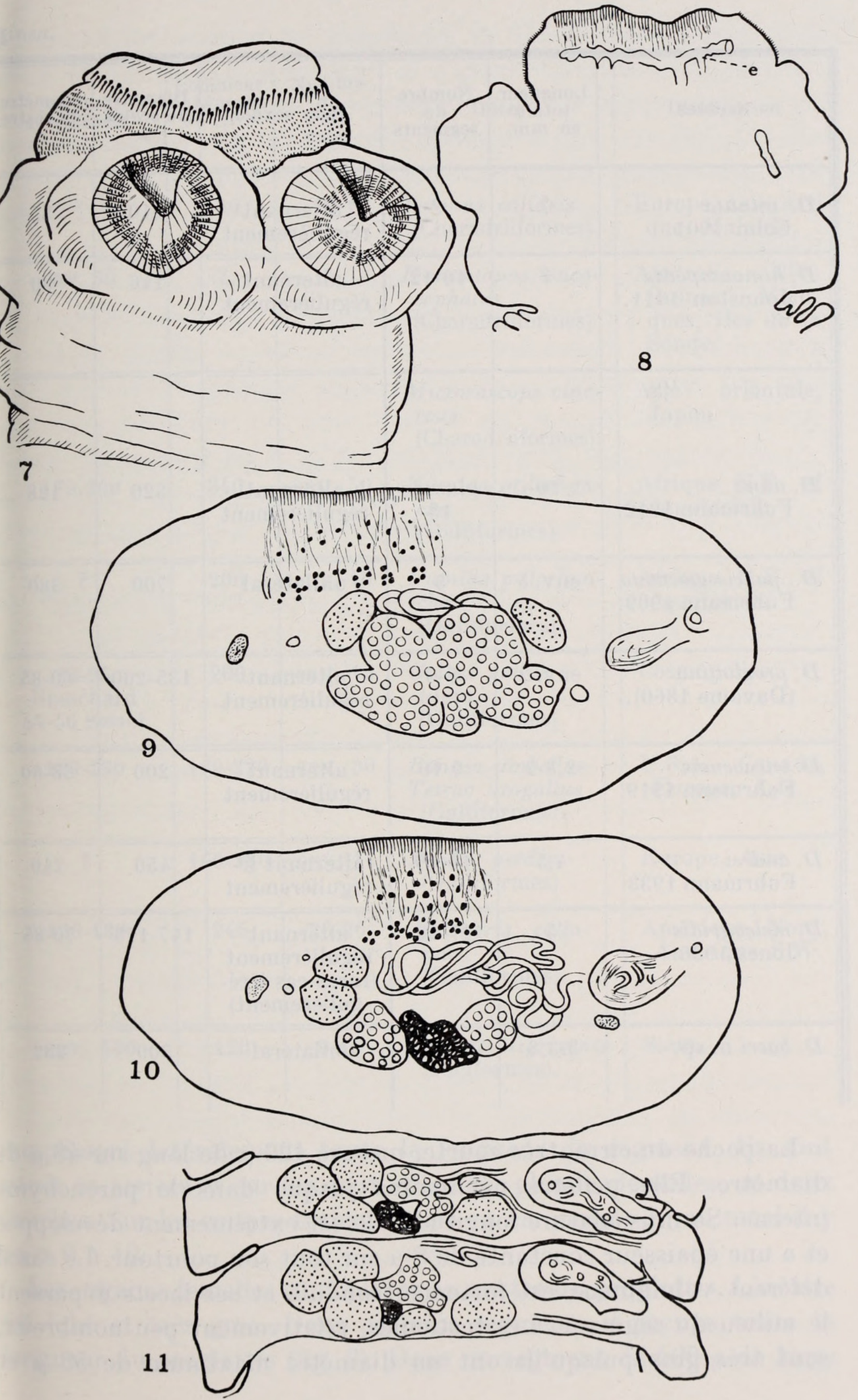




\begin{tabular}{|c|c|c|c|c|c|}
\hline Espèces & $\begin{array}{l}\text { Longueur } \\
\text { totale } \\
\text { en mm. }\end{array}$ & $\begin{array}{l}\text { Nombre } \\
\text { de } \\
\text { segments }\end{array}$ & Pore sexuel & $\begin{array}{l}\text { Diamètre } \\
\text { du scolex } \\
\mu\end{array}$ & $\begin{array}{l}\text { Diamètre } \\
\text { du rostre } \\
\mu\end{array}$ \\
\hline $\begin{array}{l}\text { D. minuta } \\
\text { Cohn } 1901 .\end{array}$ & $?$ & $?$ & $\begin{array}{l}\text { alternant } \\
\text { régulièrement }\end{array}$ & 210 & 55 \\
\hline $\begin{array}{l}\text { D. himantopodis } \\
\text { Johnston } 1911 \text {. }\end{array}$ & $?$ & $10-12$ & $\begin{array}{l}\text { alternant } \\
\text { régulièrement }\end{array}$ & 146 & 30 \\
\hline $\begin{array}{l}\text { D. nana } \\
\text { Fuhrmann } 1912 .\end{array}$ & 10 & $\begin{array}{l}\text { plus de } \\
13\end{array}$ & $\begin{array}{l}\text { alternant } \\
\text { régulièrement }\end{array}$ & 320 & 128 \\
\hline $\begin{array}{l}\text { D. paucisegmentata } \\
\text { Fuhrmann } 1909 .\end{array}$ & env. 5 & 5 & unilatéral & 700 & 380 \\
\hline $\begin{array}{l}\text { D. proglottina } \\
\text { (Davaine } 1860) \text {. }\end{array}$ & env. 2 & $5-6$ & $\begin{array}{l}\text { alternant } \\
\text { régulièrement }\end{array}$ & $135-200$ & $60-85$ \\
\hline $\begin{array}{l}\text { D. tetraoensis } \\
\text { Fuhrmann } 1919 .\end{array}$ & $2,3-3$ & $9-10$ & $\begin{array}{l}\text { alternant } \\
\text { régulièrement }\end{array}$ & 200 & $68-80$ \\
\hline $\begin{array}{l}\text { D. andrei } \\
\text { Fuhrmann } 1933 .\end{array}$ & 4,5 & $16-19$ & $\begin{array}{l}\text { alternant ir- } \\
\text { rẹ́gulièrement }\end{array}$ & 450 & 240 \\
\hline $\begin{array}{l}\text { D. meleagridis } \\
\text { Jones } 1936 .\end{array}$ & 5 & $17-22$ & $\begin{array}{l}\text { alternant } \\
\text { régulièrement } \\
\text { (rares cas irré- } \\
\text { gulièrement) }\end{array}$ & $147-175$ & $70-85$ \\
\hline D. baeri n. sp. & $3-3,6$ & $27-37$ & unilatéral & 300 & 232 \\
\hline
\end{tabular}

La poche du cirre, très courte, mesure $120 \mu$ de long sur $48 \mu \mathrm{d}$ diamètre. Elle pénètre néanmoins jusque dans le parenchym interne. Sa musculature longitudinale est extrêmement développé et a une épaisseur constante de $8 \mu$ sur tout son pourtour. Le cana déférent volumineux est fortement ondulé et ses lacets dépassen le milieu du segment. Les testicules, relativement peu nombreux sont très gros puisqu'ils ont un diamètre maximum de $56 \mu \mathrm{e}$ 


\begin{tabular}{|c|c|c|c|c|}
\hline $\begin{array}{c}\text { Nombre } \\
\text { de crochets }\end{array}$ & $\begin{array}{c}\text { I.ongueur } \\
\text { dela poche } \\
\text { du cirre } \\
\mu\end{array}$ & $\begin{array}{l}\text { Nombre } \\
\text { de } \\
\text { testi- } \\
\text { cules }\end{array}$ & Hôtes & Distribution \\
\hline$?$ & 160 & $10-12$ & $\begin{array}{l}\text { Totanus calidris } \\
\text { (Charadriiformes). }\end{array}$ & $\begin{array}{l}\text { Europe, Asie, Afri- } \\
\text { que, Malaisie. }\end{array}$ \\
\hline env. 50 & $?$ & 4 & $\begin{array}{l}\text { Himantopus leuco- } \\
\text { cephalus } \\
\text { (Charadriformes) }\end{array}$ & 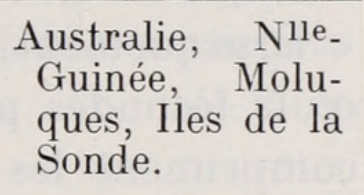 \\
\hline & & & $\begin{array}{l}\text { Microsarcops cine- } \\
\text { reus } \\
\text { (Charadriiformes). }\end{array}$ & $\begin{array}{l}\text { Asie orientale, } \\
\text { Japon. }\end{array}$ \\
\hline $170-200$ & 340 & env. 50 & $\begin{array}{l}\text { Numida ptilorhyn- } \\
\text { cha } \\
\text { (Galliformes). }\end{array}$ & $\begin{array}{l}\text { Afrique N.E. et } \\
\text { équatoriale. }\end{array}$ \\
\hline$?$ & 200 & env. 40 & $\begin{array}{l}\text { Numida ptilorhyn- } \\
\text { cha } \\
\text { (Galliformes). }\end{array}$ & $\begin{array}{l}\text { Afrique N.E. et } \\
\text { équatoriale. }\end{array}$ \\
\hline $\begin{array}{c}80-95 \\
\text { Blanchard } \\
44-50 \text { Sweet }\end{array}$ & 200 & env. 19 & $\begin{array}{l}\text { Gallus gallus } \\
\text { (dom.) } \\
\text { (Galliformes). }\end{array}$ & Cosmopolite. \\
\hline $120-130$ & $180-220$ & env. 30 & $\begin{array}{l}\text { Bonasa umbellus } \\
\text { Tetrao urogallus } \\
\text { (Galliformes). }\end{array}$ & $\begin{array}{l}\text { U.S.A., Canada, } \\
\text { Europe, Asie. }\end{array}$ \\
\hline$?$ & $160-180$ & env. 14 & $\begin{array}{l}\text { Perdix perdix } \\
\text { (Galliformes). }\end{array}$ & Europe, Asie. \\
\hline $100-130$ & 245 & $20-26$ & $\begin{array}{l}\text { Meleagris gallo- } \\
\text { pavo } \\
\text { (Galliformes). }\end{array}$ & $\begin{array}{c}\text { Amérique Nord, } \\
\text { Amérique S.W. }\end{array}$ \\
\hline env. 400 & 120 & $9-10$ & $\begin{array}{l}\text { Gecinus nigrigenis } \\
\text { (Piciformes). }\end{array}$ & Siam. \\
\hline
\end{tabular}

remplissent latéralement toute la longueur du segment. Ils sont situés en arrière et sur les deux côtés des glandes femelles, en deux couches. Sur les coupes transversales on en compte 5 ; au total il y en a 9 à 10.

Les organes femelles sont placés au centre du proglottis. L'ovaire est lobé et très gros. Dans certains segments il occupe toute la longueur du proglottis (fig. 9). Dans un segment de $500 \mu$ de large 
il a un diamètre de $172 \mu$. La glande vitellogène, logée derrière l'ovaire, a un diamètre de $65 \mu$ à $70 \mu$ dans un segment de $508 \mu$ de large. Le vagin, à paroi épaisse, passe en arrière de la poche du cirre et présente un petit réceptacle séminal presque médian et à paroi très mince. Un peu avant de se dilater en réceptacle, le vagin se rétrécit considérablement, empêchant ainsi sans doute le reflux des spermatozoïdes.

Brusquement, d'un segment à l'autre, l'ovaire disparaît et les œufs fécondés passent dans le parenchyme. Les capsules utérines compriment les vaisseaux excréteurs de même que la poche du cirre qui subsiste jusque dans le dernier segment et qui est alor: appliqué parallèlement au bord latéral du proglottis. Chaque capsule ne contient qu'un seul œuf. Il ne nous a pas été possible de mesurer les oncosphères, leur développement n'étant pas complet. Les capsules utérines mesurent $24 \mu$ à $32 \mu$ et les œuf $18 \mu$ à $20 \mu$.

A n o malie: En étudiant un grand nombre d'individus or rencontre, par-ci par-là, quelques rares coupes présentant un vagut dédoublement des organes. En effet, sur certaines coupes transver sales on trouve du côté antiporal une seconde poche du cirre rudi mentaire. Si les pores génitaux étaient régulièrement alternant, or pourrait supposer qu'il s'agit d'une coupe légèrement oblique e passant par deux proglottis simultanément, mais tel n'est justemen pas le cas.

D iscus sion: La liste comparative des espèces du genr Davainea que nous donnons à la page 154 prouve suffisammen que Davainea baeri n. sp. est une nouvelle espèce. D'abord il s'agi d'un Davainea trouvé dans un Piciforme alors que jusqu'à ce jou on n'en a rencontré que chez les Charadriiformes et les Galliformes Par son atrium génital unilatéral, la forme et le grand diamètre d son rostre et par le diamètre des ventouses, Davainea baeri s rapproche de D. paucisegmentata Fuhrmann (des Galliformes) mais il s'en éloigne considérablement par la grande différence d nombre des segments et des testicules. Seule D. andrei Fuhrman (des Galliformes) pourrait prêter à confusion, surtout si l'on tien compte de la ressemblance anatomique (ovaire large de $160 \mu$ glanse vitellogène de $64 \mu$, testicules de $60 \mu$ de diamètre). Il es regrettable que Funrmann (1933) n'ait pas pu indiquer le nombr 
t la longueur des crochets. Cependant $D$. andrei, quoique plus long jue notre espèce, a un nombre de segments beaucoup plus petit, a poche du cirre est sensiblement plus longue et ses pores génitaux ont irrégulièrement alternants. D'autre part, D. andrei n'a amais été trouvé chez un Piciforme, mais uniquement chez les Galliformes.

Pour toutes les autres espèces, Davainea baeri se range nettement i part, grâce au nombre exceptionnel des segments et de ses crochets t à l'unilatéralité des pores sexuels.

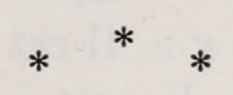

Nous décrivons ci-dessous deux nouvelles espèces de Raillietina (Raillietina) de Piciformes. Chez cet important groupe d'Oiseaux on connaissait déjà $R$.(R.) comitata (Ransom 1909) R.(R.) frontina Dujardin 1845), R.(R.) lutzi (Parona 1901) et R.(R.) pici Yamaguti 1935. En page 164 nous donnons le tableau, comparatif les espèces du genre Raillietina (Raillietina) trouvées jusqu'à ce our chez les Piciformes.

\section{Raillietina (Raillietina) fausti $n$. sp.}

Hôte: Gecinus canus (Gm.) (Piciformes).

Localité: Shansi (Chine).

Tous nos individus sont plus ou moins fragmentés et il nous est impossible d'indiquer la longueur totale. Par contre, la largeur maxima atteint $1 \mathrm{~mm}$., parfois très légèrement plus. Derrière le scolex nous avons un cou, zone non segmentée de $330 \mu$ à $350 \mu$ de long et de $220 \mu$ de large. Le premier segment est large de $260 \mu$.

Le scolex a une largeur de $300 \mu$. Les ventouses, au nombre de quatre, sont légèrement ovales et ont un diamètre de $148 \mu \times 120 \mu$; elles sont armées chacune de 8 à 10 rangs de petits crochets. Le rostre a un diamètre de $150 \mu$ à $180 \mu$. Il porte une double couronne 
de 240 à 250 crochets. La différence de longueur entre les crochets du rang antérieur et ceux du rang postérieur est très mirime. En effet, les premiers mesurent $9 \mu$ de long et les seconds $8 \mu$.

$\mathrm{Système} \mathrm{musculaire:} \mathrm{La} \mathrm{musculature} \mathrm{longitudinale}$ est divisée en deux couches assez distinctes. La couche interne forme des faisceaux très nettement délimités, tandis que les faisceaux de la couche externe se confondent presque les uns avec les autres. La musculature dorso-ventrale assez fournie n'a rien de particulier, de même que la musculature transversale.

$\mathrm{S}$ y s t è m e n e r ve u $\mathrm{x}$ : Ne présente rien de particulier.

$\mathrm{S}$ y $\mathrm{s} t$ è $\mathrm{m}$ e e $\mathrm{x}$ c r ét e u $\mathrm{r}$ : Il est composé de deux vaisseaux longitudinaux ventraux d'un diamètre de $36 \mu$ à $48 \mu$, deux vais. veaux longitudinaux dorsaux de $8 \mu$. Outre le vaisseau transverse ventral qui a un diamètre de $16 \mu$, nous avons, nettement visible un petit canal transverse dorsal de 1,7 $\mu$ de diamètre.

Organes reproducteurs: L'atrium génital, unila téral, profond de $28 \mu$, débouche dans la moitié antérieure du borc latéral du proglottis. La poche du cirre est longue de $72 \mu$ avec ur diamètre maximum de $36 \mu$. Le canal déférent est très long. Su les coupes horizontales il occupe presque toute la largeur du paren chyme interne jusqu'aux vaisseaux excréteurs antiporaux; su les coupes transversales il remplit toute la hauteur du parenchym interne. Il est entouré de grosses cellules prostatiques sur tout sol parcours. Au centre du segment il n'a que $8 \mu$ de diamètre, pui il s'élargit considérablement, atteignant un diamètre de $20 \mu$ à 24 । et joue ainsi le rôle de vésicule séminale externe. Peu avant d pénétrer dans la poche du cirre, il se rétrécit de nouveau à enviro] $5 \mu$ à $6 \mu$. A l'intérieur de la poche du cirre il mesure $12 \mu$ de largc Le cirre est long de $45 \mu$ et a un diamètre de $2 \mu$.

Les testicules sont au nombre de 18 à 20, dont à peu prè 6 poraux, 1 à 3 médians et 11 à 12 antiporaux. Ils sont légèremen ovales, d'un diamètre de $68 \mu$ à $72 \mu \times 48 \mu$ à $56 \mu$.

Le vagin débouche dans l'atrium en arrière de la poche du cirr Son diamètre est d'abord de $3,5 \mu$ à $6 \mu$, puis il se dilate en $u$ réceptacle séminal énorme, fortement ondulé, long de $170 \mu$ à $180 \mu$ avec un diamètre de $20 \mu$. Après le réceptacle, le vagin se rétréci légèrement puis augmente de nouveau jusqu'à un diamètre d'er viron $10 \mu$. A cet endroit il s'incurve brusquement dorso-ventrc lement et chemine vers l'isthme ovarien. 

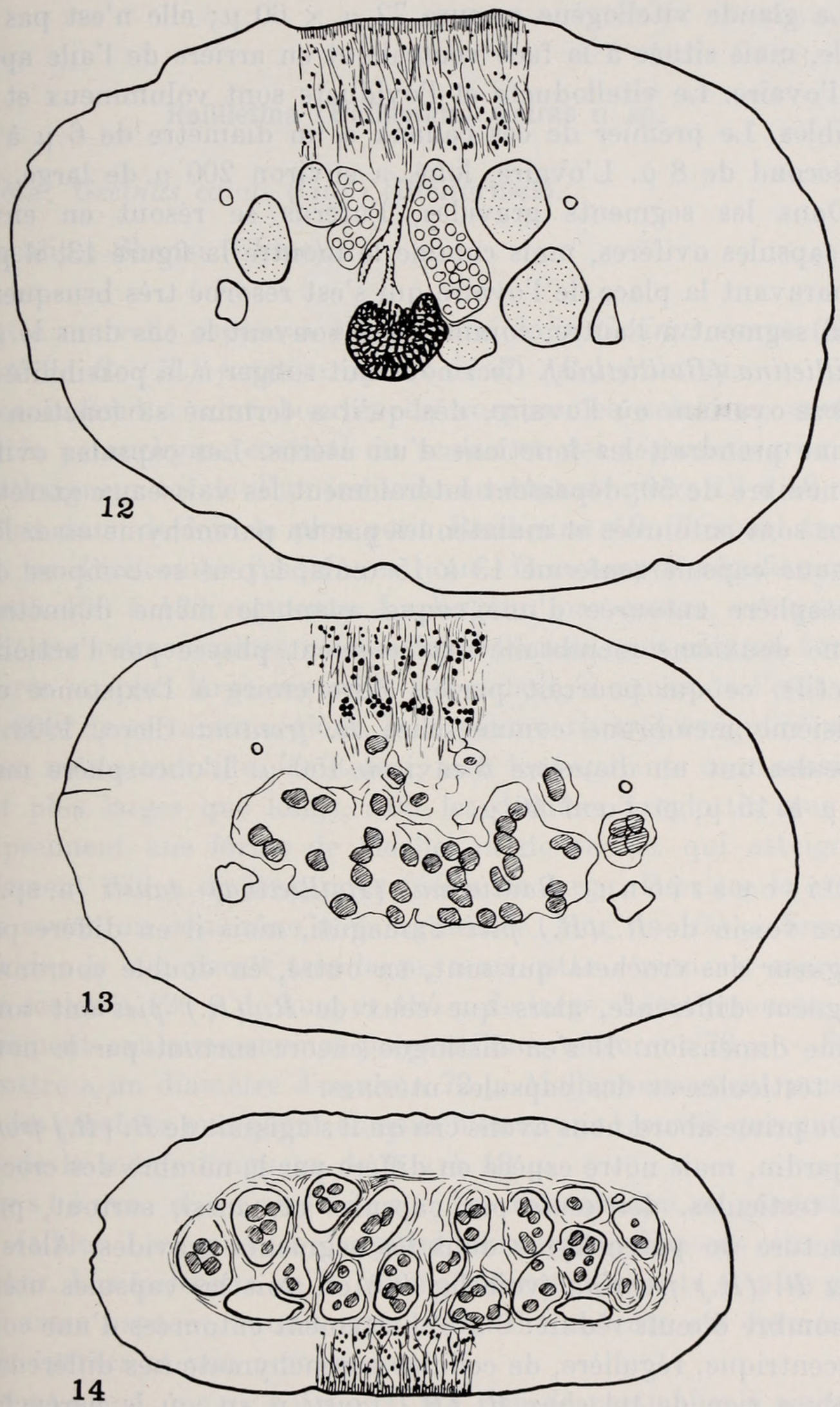

FIG. 12-14.

Raillietina (Raillietina) fausti n. sp.: 12. coupe transversale passant par les organes femelles; 13. utérus jeune, en coupe transversale; 14. coupe transversale à travers un segment gravide montrant les capsules ovifères.

Rev. Suis : e de Zool., T. 48, 1941. 
La glande vitellogène mesure $72 \mu \times 60 \mu$; elle n'est pas cen trale, mais située à la face ventrale et en arrière de l'aile aporal de l'ovaire. Le vitelloducte et l'oviducte sont volumineux et bie: visibles. Le premier de ces canaux a un diamètre de $6 \mu$ à $7 \mu$ le second de $8 \mu$. L'ovaire, lobé, a environ $200 \mu$ de large.

Dans les segments gravides, l'utérus se résout en enviro 50 capsules ovifères, mais comme le montre la figure 13 , il pren auparavant la place de l'ovaire qui s'est résorbé très brusquemen d'un segment à l'autre, comme c'est souvent le cas dans le genr Raillietina (Raillietina). Ceci nous fait songer à la possibilité d'u utérus-ovarium où l'ovaire, dès qu'il a terminé sa fonction ové rienne prendrait les fonctions d'un utérus. Les capsules ovifères au nombre de 50, dépassent latéralement les vaisseaux excréteur: Elles sont entourées et maintenues par un parenchyme assez lâch Chaque capsule renferme 13 à 15 œufs. L'œuf se compose d'un oncosphère entourée d'une coque ayant le même diamètre, $\epsilon$ d'une deuxième membrane extrêmement plissée par l'action de réactifs, ce qui pourrait parfois faire croire à l'existence d'un troisième membrane comme chez $R$. frontina Clerc, 1903. Lt capsules ont un diamètre d'environ $150 \mu$. L'oncosphère mesur $14 \mu$ à $15 \mu$, et l'œuf $40 \mu$.

D iscussion: Raillietina (Raillietina) fausti n. sp. e: assez voisin de $R$. (R.) pici Yamaguti, mais il en diffère par longueur des crochets qui sont, en outre, en double couronne $c$ longueur différente, alors que ceux de $R$. (R.) pici ont tous même dimension. Il s'en distingue encore surtout par le nombs des testicules et des capsules utérines.

De prime abord nous avons cru qu'il s'agissait de $R$. (R.) frontir Dujardin, mais notre espèce en diffère par le nombre des crochet des testicules, des œufs par capsules et, aussi, surtout, par structure du parenchyme dans les segments gravides. Alors qu chez R. (R.) frontina (voir fig. 20) on voit les capsules utérin (à nombre d'œufs réduit: 6 à 8 ) nettement entourées d'une coucl concentrique, régulière, de cellules parenchymateuses différenciée il n'y a rien de tel chez $R$. (R.) fausti n. sp. où le parenchyn intercapsulaire est non différencié. 


\section{Raillietina (Raillietina) clairae n. sp.}

Hôte: Gecinus canus (Gm.) (Piciformes).

Localité: Shansi (Chine).

Cette nouvelle espèce a été trouvée dans Gecinus canus (Gm.) à côté de $R$. (R.) fausti n. sp. et de $R$. (R.) frontina.

Nos individus sont bourrés de corpuscules calcaires, surtout dans le parenchyme cortical du scolex et des segments gravides.

La longueur totale d'un individu ne dépasse guère 12 à $18 \mathrm{~mm}$. et c'est donc de loin le plus petit Raillietina (Raillietina) trouvé chez les Piciformes jusqu'à ce jour. On compte régulièrement environ 100 à 120 segments. La largeur, par contre, est pareille à celle des autres espèces, c'est-à-dire $0,95 \mathrm{~mm}$., soit environ $1 \mathrm{~mm}$., mesurée au plus large segment. Cependant, à cause de l'état très contracté de certaines régions, la largeur varie très irrégulièrement sur un même individu. Toutefois, tous les segments sont sensiblement plus larges que longs, sauf les derniers proglottis gravides qui prennent une forme de cloche ou de sac et qui atteignent finalement $630 \mu$ de long pour $650 \mu$ de large. Derrière le scolex nous avons un cou, zône non segmentée, long de $160 \mu$. Sur nos individus le cou paraît très large, mais cette région est macérée. Le scolex a $120 \mu$ de long et $184 \mu$ de large. Les ventouses, très légèrement ovalaires sur nos préparations, mesurent $76 \mu \times 68 \mu$. Le rostre a un diamètre d'environ $72 \mu$. Malheureusement presque tous les crochets manquent et nous n'en avons trouvés que quatre, tous de la même longueur de $9 \mu$ à $10 \mu$.

$\mathrm{S}$ y s t è $\mathrm{me} \mathrm{m} \mathrm{u} \mathrm{s} \mathrm{c} \mathrm{u} \mathrm{l} \mathrm{a} \mathrm{i} \mathrm{re}$ : La musculature en général est très faible. Les muscles longitudinaux sont disposés en deux couches. Les faisceaux de la couche interne, distincts et plus gros, sont assez espacés, tandis que la couche externe est formée de fibres éparses et peu nombreuses.

S y s t è m e n e r ve u x : Il n'a rien de particulier à signaler. S y s t èm e e x c r ét e u r : Le diamètre des vaisseaux excréteurs ventraux qui est de $12 \mu$ à $13 \mu$ dans les segments adultes, atteint $20 \mu$ à $25 \mu$ dans les segments gravides. Les vaisseaux 
dorsaux ont un diamètre de $2,5 \mu$ à $3 \mu$. Les deux vaisseaux ven traux sont reliés, dans la région postérieure du proglottis, par u petit vaisseau transversal.

S y s t è m e r e p r o d u c t e u r : L'atrium génital, unilatéral débouche dans le quart antérieur du bord latéral du proglottis La poche du cirre est longue de $48 \mu$ à $60 \mu$. Son diamètre est $d$ $28 \mu$. Le canal déférent est long et traverse presque tout le paren chyme interne. Contrairement à ce qu'on observe dans la règl générale, il est presque rectiligne dans toute sa moitié porale e c'est au milieu du segment, au niveau de l'ovaire, qu'il ondule e grands lacets. Son diamètre est de $6 \mu$ à $7 \mu$. A l'intérieur de 1 poche du cirre ce diamètre ne diminue que très peu et attein $5 \mu$ à $5,5 \mu$. Le cirre a un diamètre de 1,7 $\mu$. Il n'y a pas de vésicule séminales, ni interne, ni externe.

Les testicules, au nombre de 20 environ, entourent les glande femelles. On en compte 6 à 7 du côté poral, 3 à 4 postérieuremen en arrière de l'ovaire et de la glande vitellogène, et 9 à 10 du côt antiporal. Leur diamètre est de $40 \mu$.

Le vagin, d'abord d'un diamètre de $5 \mu$, longe la poche du cirı sur une distance de $50 \mu$ à peu près, puis il se rétrécit jusqu'à u diamètre de $2,5 \mu$ sur une longueur de $25 \mu$ pour se dilater ensuit en un réceptacle séminal fusiforme mesurant $60 \mu \times 20 \mu$. ( réceptacle n'est donc pas plus ou moins médian, mais se trour situé derrière la portion rectiligne du canal déférent et débute a niveau de l'extrémité interne de la poche du cirre.

L'ovaire, médian, forme une masse compacte légèrement lobé d'une largeur de $145 \mu$ à $160 \mu$ dans les segments adultes. C organe disparaît brusquement, laissant les œufs épars dans parenchyme. Il n'y a pas d'utérus à proprement parler. Ce derni se résout en 20 à 30 capsules utérines. La glande vitellogène $80 \mu$ de large et $52 \mu$ de long. Elle se trouve en arrière de l'ovaiı

\section{FIG. 15-19.}

Raillietina (Raillietina) clairae n. sp.: 15. segment adulte; 16. œufs disper: dans le parenchyme; 17 . capsules ovifères en voie de formation; 18 . co sules ovifères formées; 19. coupe transversale d'un segment gravide.

FIG. 20.

Raillietina (Raillietina) frontina (Clerc): coupe transversale d'un segme: gravide d'après le matériel original. 
Espèces du genre

\begin{tabular}{|c|c|c|c|c|c|c|}
\hline Espèces & $\begin{array}{l}\text { Longueur } \\
\text { en mm. }\end{array}$ & $\begin{array}{l}\text { Largeur } \\
\text { en mm. }\end{array}$ & $\begin{array}{c}\text { Diamètre } \\
\text { du } \\
\text { scolex } \\
\mu\end{array}$ & $\begin{array}{c}\text { Diamètre } \\
\text { du } \\
\text { rostre } \\
u\end{array}$ & $\begin{array}{c}\text { Diamètre } \\
\text { des } \\
\text { ventouses } \\
\mu\end{array}$ & $\begin{array}{l}\text { Nombre } \\
\text { de } \\
\text { crochets }\end{array}$ \\
\hline $\begin{array}{l}\text { R. (R.) comi- } \\
\text { tata (Ransom } \\
\text { 1909). }\end{array}$ & $45-55$ & 1,16 & $250-290$ & 90 & $\begin{array}{c}160 \times \\
115-135\end{array}$ & $\begin{array}{l}\text { env. } 80 \\
\text { couronne } \\
\text { simple. }\end{array}$ \\
\hline $\begin{array}{l}R . \text { (R.) frontina } \\
\text { (Dujardin } \\
\text { 1845). }\end{array}$ & $50-80$ & $1-1,4$ & $\begin{array}{c}420 \\
(230 \\
\text { FUHR- } \\
\text { MANN) }\end{array}$ & $80-110$ & $160-200$ & 180 \\
\hline $\begin{array}{l}\text { R. } \\
\text { (Parona lutzi } 1901) .\end{array}$ & 60 & 1 & 470 & 70 & 110 & env. 100 \\
\hline $\begin{array}{l}\text { R. }(R .) \text { pici } \\
\text { Yamaguti } \\
1935 .\end{array}$ & $\begin{array}{c}36 \\
250 \\
\text { segments }\end{array}$ & 0,93 & 330 & $100-150$ & 100 & $?$ \\
\hline $\begin{array}{l}\text { R. (R.) fausti } \\
\text { n. sp. }\end{array}$ & $?$ & 1 et plus & 300 & $150-180$ & $148 \times 120$ & $240-250$ \\
\hline $\begin{array}{l}R . \text { (R.) clairae } \\
\text { n. sp. }\end{array}$ & $\mid \begin{array}{c}12-18 \\
100 \text { à } 120 \\
\text { segments }\end{array}$ & 0,95 & 184 & 72 & $76 \times 68$ & $?$ \\
\hline
\end{tabular}

mais il n'est pas rare qu'au lieu d'être médiane, elle soit situér du côté poral de l'ovaire.

Comme le montrent les figures 16, 17, 18, le groupement de œufs en capsules ovifères se fait petit à petit. D'abord, sur un distance de 8 segments environ, les œeufs sont isolés, épars, dan un parenchyme à noyaux vivement colorés. Au fur et à mesur que le segment mûrit, les œufs se groupent, les cellules parenchy mateuses se différencient et se condensent autour de ces groupe d'œufs. On compte environ 4 segments ayant cet aspect. Finale ment, dans les segments gravides, on trouve des capsules défini tivement formées par une enveloppe de cellules différenciées. l'intérieur d'une capsule, les œufs sont encore entourés de cellule 
) des Piciformes.

\begin{tabular}{|c|c|c|c|c|c|}
\hline $\begin{array}{l}\text { Nombre } \\
\text { de } \\
\text { testi- } \\
\text { cules }\end{array}$ & $\begin{array}{l}\text { Nombre } \\
\text { de } \\
\text { capsules } \\
\text { utérines }\end{array}$ & $\begin{array}{l}\text { Geufs } \\
\text { par } \\
\text { capsules }\end{array}$ & Remarque & Hôtes & $\begin{array}{l}\text { Distri- } \\
\text { bution }\end{array}$ \\
\hline $30-35$ & $40-50$ & $6-12$ & $\begin{array}{l}\text { Capsules ne } \\
\text { dépassant } \\
\text { pas les vais- } \\
\text { seaux excré- } \\
\text { teurs. }\end{array}$ & $\begin{array}{l}\text { Colaptes auratus, } \\
\text { Melonerpes } \\
\text { erythrocephalus. }\end{array}$ & $\begin{array}{l}\text { Amérique } \\
\text { du Nord. }\end{array}$ \\
\hline $\begin{array}{l}15-16 \\
(\text { CLERC } \\
50)\end{array}$ & $35-40$ & $\begin{array}{c}6-8 \\
\text { (CLERC) }\end{array}$ & $\begin{array}{l}\text { Capsules dé- } \\
\text { passant les } \\
\text { vaisseaux ex- } \\
\text { créteurs. }\end{array}$ & $\begin{array}{l}\text { Colaptes campes- } \\
\text { tris, Dendroco- } \\
\text { pus major, Ge- } \\
\text { cinus viridis, } \\
\text { Picus martius, } \\
\text { Picus spec. }\end{array}$ & $\begin{array}{l}\text { Cosmopo- } \\
\text { lite. }\end{array}$ \\
\hline$?$ & $12-16$ & $?$ & - & $\begin{array}{l}\text { Celeus flavescens, } \\
\text { Picus spec. }\end{array}$ & $\begin{array}{l}\text { Amérique } \\
\text { du Sud. }\end{array}$ \\
\hline 30 & env. 20 & $\begin{array}{c}12 \\
\text { ou plus }\end{array}$ & - & $\begin{array}{l}\text { Picus awokera } \\
\text { awokera. }\end{array}$ & Asie. \\
\hline $18-20$ & env. 50 & $13-15$ & $\begin{array}{l}\text { Capsules dé- } \\
\text { passant les } \\
\text { vaisseaux } \\
\text { excréteurs. }\end{array}$ & Gecinus canus. & Chine. \\
\hline env. 20 & $20-30$ & $12-14$ & $\begin{array}{l}\text { Capsules ne } \\
\text { dépassant } \\
\text { pas les vais- } \\
\text { seaux excré- } \\
\text { teurs. }\end{array}$ & Gecinus canus. & Chine. \\
\hline
\end{tabular}

parenchymateuses, mais ces dernières sont en voie de dégénérescence granulomateuse et les granulations dont elles sont bourrées se colorent presque en noir à l'hémalun. Sur les coupes horizontales on aperçoit un parenchyme intercapsulaire sous forme d'un réseau très lâche et ne se colorant pour ainsi dire pas (voir fig. 18). Les capsules ovifères ne dépassent pas latéralement les vaisseaux excréteurs; elles les compriment tout au plus un peu (fig. 19). Il y a done 20 à 30 capsules parenchymateuses contenant chacune 12 à 14 œufs. Les capsules ont un diamètre d'environ $140 \mu$. L'œuf mesure $25,5 \mu$. L'oncosphère a un diamètre de $12 \mu$ et est entourée d'une membrane très variable et fortement plissée, mesurant $17 \mu$ à $25 \mu$. 
D is c u s s i o n : Raillietina (Raillietina) clairae n. sp. ne peut être confondu avec aucune des cinq autres espèces de notre liste comparative de la page 00 . Il s'en distingue immédiatement par son strobila très court à nombre restreint de proglottis, son scolex proportionnellement étroit, portant également des ventouses très petites. S'il se rapproche de $R$. (R.) pici par la longueur des crochets et le nombre des œufs par capsule, il s'en éloigne, par contre, par le nombre des testicules et des capsules ovifères.

\section{Raillietina (Paroniella) siamensis n. sp.}

\section{Hôtes: Thereiceryx lineatus (Vieill.) Thereiceryx phaeostriatus (Bp.) \\ (Capitoniformes).}

Localité: Siam.

Ainsi que le montre la figure 21 , nos individus sont presque tous dans un état de contraction extrême. Ceci a naturellement donné lieu à quelques difficultés dans l'étude et la mensuration de certains organes.

La longueur totale d'un individu est d'environ $45 \mathrm{~mm}$. pour une largeur maxima de 4,8 à $4,9 \mathrm{~mm}$.

Derrière le scolex nous avons un cou, zône non segmentée, dont la longueur varie énormément selon l'état de contraction de l'individu. Nous avons trouvé, en effet, des individus avec un cou de $220 \mu$ de long, un autre de $660 \mu$ et un troisième qui atteignait même $770 \mu$.

FIG. 21-23.

Raillietina (Paroniella) siamensis n. sp.: 21. coupe horizontale d'un segment adulte; 22. coupe transversale de la région porale; 23. portion d'une coupe à travers un segment gravide montrant les corpuscules calcaires et les cellules parenchymateuses.

FIG. 24-26.

Raillietina (Paroniella) pinsonae n. sp.: 24. coupe transversale montrant le testicules; 25 . coupe transversale à travers l'ovaire et la région porale 26. portion d'une coupe transversale à travers un segment gravide montrant les cellules glandulaires. 


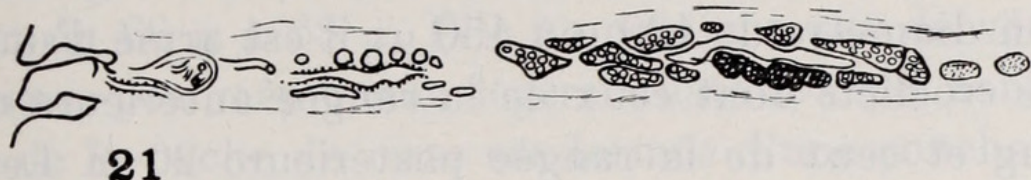
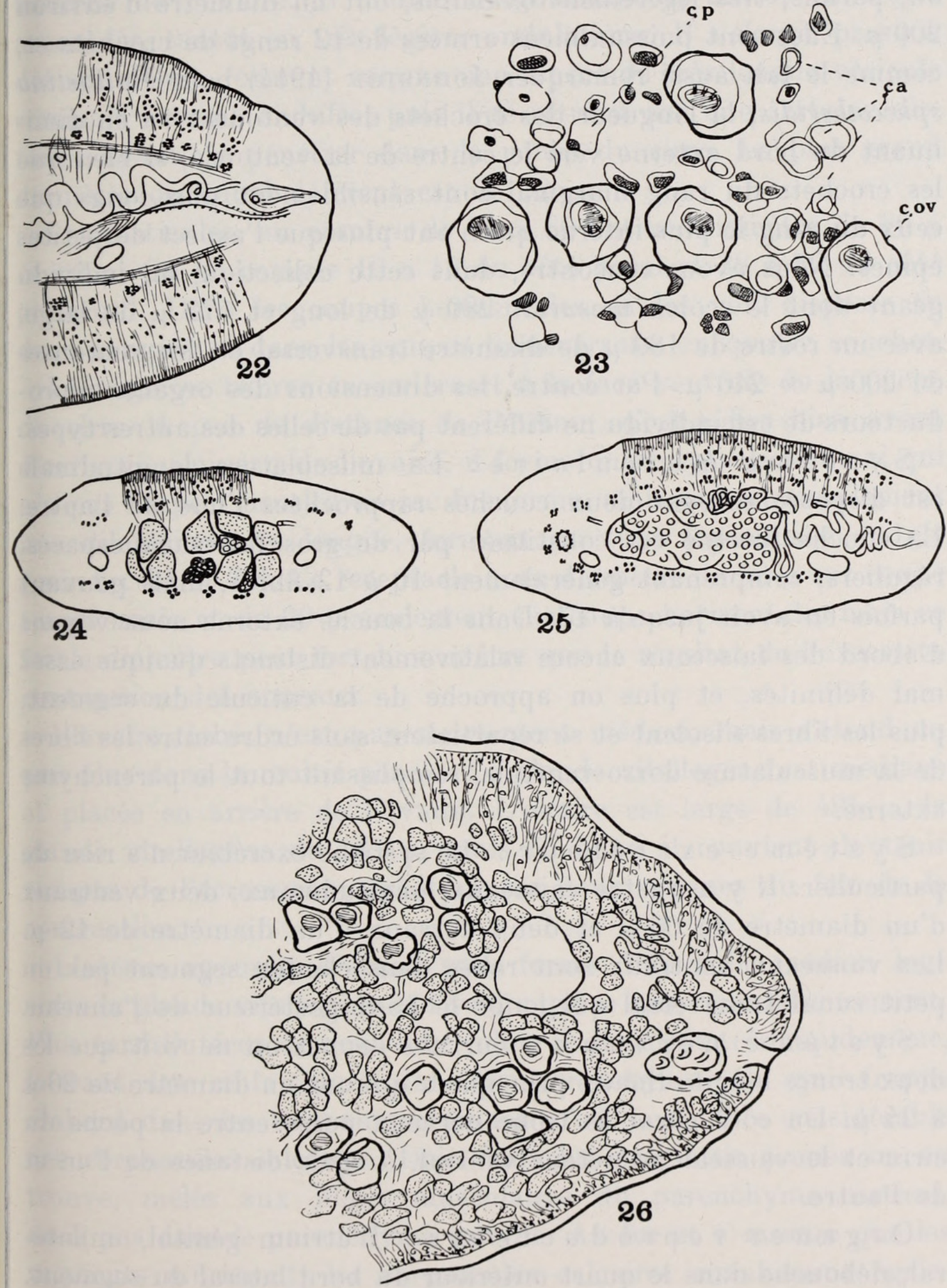
Le scolex a $240 \mu$ de long et $400 \mu$ à $440 \mu$ de large. Le rostre, arrondi, a un diamètre de $130 \mu$ à $150 \mu$; il est armé d'une double couronne de crochets dont ceux de la rangée antérieure mesurent $24 \mu$ de long et ceux de la rangée postérieure $20 \mu$. Le nombre total de ces crochets est d'environ 240. Les ventouses, circulaires ou, parfois, très légèrement ovalaires, ont un diamètre d'environ $200 \mu$. Elles sont puissamment armées de 12 rangs de crochets et, comme le fait aussi remarquer Jонnston (1914) pour Raillietina sphecotheridis, la longueur des crochets des ventouses va en diminuant du bord externe vers le centre de la ventouse, si bien que les crochets du rang marginal sont sensiblement plus longs que ceux du rang le plus interne qui n'ont plus que l'aspect de petites épines. Nous avons rencontré, dans cette collection, un individu géant dont le scolex mesurait $280 \mu$ de long et $572 \mu$ de large, avec un rostre de $180 \mu$ de diamètre transversal et des ventouses de $200 \mu \times 240 \mu$. Par contre, les dimensions des organes reproducteurs de cet individu ne diffèrent pas de celles des autres types.

S y stè m e m u s cula i re: La musculature longitudinale est disposée suivant deux couches rapprochées l'une de l'autre. La couche interne est constituée par de gros faisceaux espacés, réguliers, comprenant généralement 10 à 12 fibres, mais pouvant parfois en avoir jusqu'à 17. Dans la couche externe nous voyons d'abord des faisceaux encore relativement distincts quoique assez mal délimités, et plus on approche de la cuticule du segment. plus les fibres s'isolent et se répartissent sans ordre entre les fibres de la musculature dorso-ventrale, remplissant tout le parenchyme externe.

S y s t è m e e x c r é t e u r : Le système excréteur n'a rien de particulier. Il y a quatre vaisseaux longitudinaux: deux ventraus d'un diamètre de $66 \mu$ et deux dorsaux d'un diamètre de $12 \mu$ Les vaisseaux ventraux sont reliés dans chaque segment par ur petit canal transversal qui longe le bord postérieur de l'anneau

Système nerveux: Sur nos coupes on ne voit que le: deux trones longitudinaux principaux qui ont un diamètre de 20 à $24 \mu$. Du côté poral, le tronc nerveux passe entre la poche di cirre et le vaisseau excréteur ventral, à égale distance de l'un e de l'autre.

Organes reproducteurs: L'atrium génital, unilaté ral, débouche dans le quart antérieur du bord latéral du segment 
La poche du cirre a $150 \mu$ à $160 \mu$ de long et un diamètre maximum de $66 \mu$ à $68 \mu$. Elle ne contient pas de vésicule séminale interne, pas même une légère dilatation du canal déférent. La paroi de la poche du cirre est formée d'une couche musculaire puissante. Le canal déférent n'est pas très long, s'arrêtant à la hauteur de l'ovaire. Il ondule irrégulièrement, tantôt en lacets accentués, tantôt en très légères ondulations. Il s'élargit presque dès son début, formant comme une espèce de très longue vésicule séminale externe ondulée, puis il se rétrécit sur une certaine distance avant de pénétrer dans la poche du cirre. Le plus grand diamètre du canal déférent est de $32 \mu$.

Les testicules, au nombre de 50 à 60 ont un diamètre de $80 \mu$ $\times 60 \mu$. On en compte 10 à 12 du côté poral et 38 à 50 du côté antiporal. Dans les coupes horizontales on les voit disposés en deux rangées et dans les coupes transversales en quatre couches.

Le vagin se trouve en arrière et à la face ventrale de la poche du cirre. A peu de distance de l'atrium, c'est-à-dire bien avant d'arriver au niveau de l'ovaire, il forme un réceptacle séminal qui n'a pas l'aspect usuel d'un sac fusiforme, mais qui est simplement une dilatation très longue, formant un tube légèrement ondulé (voir fig. 21 et 22). Ce réceptacle a un diamètre de $56 \mu$ sur coupe transversale et de $20 \mu$ seulement sur coupe horizontale, mais ce faible diamètre peut fort bien n'être que le résultat de l'excessive contraction des anneaux.

L'ovaire, lobé, n'est pas strictement médian, mais s'étend un peu plus dans la moitié porale. La glande vitellogène est médiane et placée en arrière de l'ovaire. L'ovaire est large de $495 \mu$, la glande vitellogène de $145 \mu$, mais ici aussi il convient de tenir compte de l'écrasement subi par ces deux organes du fait de la contraction.

L'utérus apparaît d'abord sous forme d'un tube médian logé entre la glande vitellogène et l'ovaire qui le couvre antérieurement. Plus tard l'utérus devient lobé, mais il disparaît très rapidement, laissant les œufs épars dans le parenchyme. Ce qui frappe d'abord dans les segments gravides, c'est le nombre considérable des corpuscules calcaires. Déjà dans les proglottis jeunes on en trouve, mêlés aux fibres musculaires du parenchyme cortical, mais pas dans le parenchyme interne. Au fur et à mesure que les segments mûrissent, les corpuscules calcaires envahissent égale- 
ment le parenchyme interne et, dans les cellules gravides, la plupart des cellules parenchymateuses se transforment en corpuscules calcaires. Dans la figure 23 on voit les capsules utérines entourées et comme fixées par la masse des corpuscules calcaires et des cellules parenchymateuses en voie de transformation. Les corpuscules calcaires atteignent jusqu'à $16 \mu$ à $18 \mu$ de diamètre. Chaque capsule ne contient qu'un seul œuf. Cet œuf est formé de l'embryon qui est entouré d'une première membrane l'enserrant d'assez près et d'une seconde membrane très grande et de forme irrégulière. Les capsules ont un diamètre de $30 \mu$ à $36 \mu$, l'embryon mesure $12 \mu$ à $14 \mu$, la première membrane $15 \mu$ à $16 \mu$ et la deuxième $26 \mu$ à $28 \mu$. Dans les segments gravides, les capsules passent latéralement au-délà des vaisseaux excréteurs et des deux troncs nerveux principaux.

D is c us sion: A première vue nous avons cru nous trouver en présence de $R$. (P.) sphecotheridis Johnston, que certains auteurs placèrent, par erreur, parmi les Cestodes des Capitoniformes, mais il est certain que le véritable $R$. (P.) sphecotheridis Johnston 1914, est un Cestode de Passeriformes puisqu'il fut trouvé chez Sphecotheres maxillaris (Lath.). De ce fait, $R$. (P.) siamensis n. sp. est le premier de ce genre rencontré chez les Capitoniformes. D'ailleurs, une étude un peu plus minutieuse et quelques dimensions prises à titre de comparaison prouvèrent rapidement qu'il ne s'agissait pas de $R$. (P.) sphecotheridis. Johnston a décrit un scolex complètement couvert de petites épines plus nombreuses, plus denses, à la base du rostre. Chez notre espèce il n'y a des épines qu'à la base du rostre et non sur tout le scolex. Il est vrai que ces épines sont très caduques, mais il serait étonnant qu'aucune n'ait subsisté sur l'un ou l'autre de nos individus. Puis Johnston décrit et dessine une poche du cirre renfermant une vésicule séminale interne. Dans notre espèce, que l'on prenne des coupes horizontales ou des coupes transversales, il n'y a jamais trace d'une vésicule séminale interne. Johnston a compté environ 30 testicules mesurant $25 \mu$ à $30 \mu \times 15 \mu$ à $20 \mu$, alors que nous en trouvons 50 à 60 mesurant $80 \mu$ à $90 \mu \times 50 \mu$ à $60 \mu$. Les crochets de $R$. (P.) sphecotheridis sont longs de $20 \mu$ et $15 \mu$, tandis que ceux de notre Cestode mesurent $24 \mu$ et $20 \mu$. Si l'on tient encore compte des différences assez notables dans la longueur et la largeur du strobila, 
dans la longueur de la poche du cirre, dans le diamètre de l'embryon, etc., il appert qu'aucune confusion n'est possible, surtout si l'on note en première ligne que l'hôte n'est pas un Passeriforme, mais un Capitoniforme.

\section{Raillietina (Paroniella) pinsonae n. sp.}

\section{Hôtes: Gecinus nigrigenis (Wardl. Rams.) Gecinus chirrolophus (Vieill.) \\ (Piciformes.)}

\section{Localité: Siam.}

Raillietina (Paroniella) pinsonae n. sp. a été trouvé côte à côte avec Davainea baeri n. sp. dans l'intestin de Gecinus nigrigenis (Wardl. Rams.). En outre nous l'avons retrouvé, dans la mème collection, chez Gecinus chirrolophus (Vieill.).

Raillietina (Paroniella) pinsonae n. sp. a une longueur totale de 18 à $19 \mathrm{~mm}$. seulement, pour une largeur maxima de $1 \mathrm{~mm}$. Derrière le scolex, le cou, zône non segmentée, a environ $100 \mu$ à $120 \mu$ de long et $240 \mu$ de large. Les premiers segments sont larges de $240 \mu$. Le plus grand proglottis trouvé sur des fragments est large de $990 \mu$ et long de $495 \mu$; c'est un segment mûr. Le dernier proglottis d'un individu complet est un peu plus long $(550 \mu)$, mais moins large $(880 \mu)$, différence due, sans doute, à la contraction.

Le scolex a environ $320 \mu$ de diamètre et une longueur de $220 \mu$. Il porte quatre ventouses légèrement ovales, mesurant longitudinalement $170 \mu$ et transversalement $140 \mu$. Elles sont armées de nombreux cercles de petits crochets longs de $5 \mu$ à $6 \mu$ et disposés en rangées obliques. Le rostre a un diamètre de $75 \mu$ à $90 \mu$. Comme nous n'avions aucun rostre évaginé, il nous a été difficile d'en mesurer la longueur, mais il semble être plutôt court, ne dépassant guère $40 \mu$ à $50 \mu$. Il est armé d'une double couronne de 160 à 180 petits crochets d'une longueur de $7 \mu$ à $8 \mu$. Cette longueur des crochets est remarquablement petite comparée à celle des autres espèces du sous-genre qui varie entre $14 \mu$ et $18 \mu$. Comme chez Davainea baeri n. sp. on remarque nettement la fibre musculaire s'attachant à chacun de ces crochets. 
Espèces du genre

\begin{tabular}{|c|c|c|c|c|c|c|}
\hline Espèces & $\begin{array}{l}\text { Longueur } \\
\text { en mm. }\end{array}$ & $\begin{array}{l}\text { Largeur } \\
\text { en mm. }\end{array}$ & $\begin{array}{c}\text { Diamètre } \\
\text { du } \\
\text { scolex } \\
\mu\end{array}$ & $\begin{array}{c}\text { Diamètre } \\
\text { des } \\
\text { ventouses } \\
\mu\end{array}$ & $\begin{array}{c}\text { Diamètre } \\
\text { du } \\
\text { rostre } \\
\mu\end{array}$ & $\begin{array}{l}\text { Nombre } \\
\text { de } \\
\text { crochets }\end{array}$ \\
\hline $\begin{array}{l}\text { R. (P.) cruciata } \\
\text { (Rudolphi } \\
\text { 1819). }\end{array}$ & $\begin{array}{c}40 \\
\text { sans } \\
\text { segments } \\
\text { tout à } \\
\text { fait mûrs }\end{array}$ & 0,8 & $300-410$ & $130-190$ & 126 & env. 200 \\
\hline $\begin{array}{c}\text { R. (P.) longis- } \\
\text { pina (Fuhr- } \\
\text { mann 1908). }\end{array}$ & 100 & 1,5 & 320 & 150 & 64 & $\begin{array}{l}\text { nom- } \\
\text { breux }\end{array}$ \\
\hline $\begin{array}{l}\text { R. (P.) rhynchota } \\
\text { (Ransom } \\
\text { 1909). }\end{array}$ & $50-60$ & 1 & $320-380$ & $150-160$ & $120-160$ & env. 400 \\
\hline $\begin{array}{l}\text { R. (P.) cirroflexa } \\
\text { Tubangui et } \\
\text { Masiluñgan } \\
1937 .\end{array}$ & 20 & 0,8 & 300 & $\begin{array}{c}100-130 \\
\times 90-120\end{array}$ & $150-200$ & 250 \\
\hline $\begin{array}{l}R .(P .) \text { pinsonae } \\
\text { n. sp. }\end{array}$ & $18-19$ & 1 & 320 & $170 \times 140$ & $75-90$ & $160-180$ \\
\hline
\end{tabular}

S y s t èm e n e rve ux: Sur nòs coupes on ne voit que les deux trones longitudinaux principaux.

$\mathrm{S} y \mathrm{~s} t$ èm e e $\mathrm{x}$ c r ét e ur: Les vaisseaux excréteurs ventraux sont gros et ont un diamètre de $44 \mu$. Les vaisseaux dorsaux. à parci plus épaisse, mesurent $12 \mu$.

Système muscula i re: La musculature longitudinale est formée de deux couches disposées en faisceaux. Les faisceaux de la couche externe, disposés en plusieurs couches, sont moins denses et moins distinctement séparés que ceux de la couche interne. La musculature dorso-ventrale, bien développée, allongє ses fibres entre les faisceaux de la musculature longitudinale.

Organes reprod u c te urs: Comme chez tous les Rail. lietina (Paroniella) les pores sexuels sont unilatéraux. Ils débou. chent dans le tiers antérieur du bord latéral du segment. 
๖) des Piciformes.

\begin{tabular}{|c|c|c|c|c|}
\hline $\begin{array}{c}\text { Nombre } \\
\text { de } \\
\text { testi- } \\
\mu\end{array}$ & $\begin{array}{l}\text { Diamètre } \\
\text { de l'onco- } \\
\text { sphère } \\
\mu\end{array}$ & Remarques & Hôtes & $\begin{array}{l}\text { Distri- } \\
\text { bution }\end{array}$ \\
\hline$?$ & 12 & $\begin{array}{l}\text { Les œufs semblent dis- } \\
\text { séminés directement } \\
\text { dans le parenchyme } \\
\text { interne }\end{array}$ & $\begin{array}{l}\text { Ceophloeus li- } \\
\text { neatus, Geci- } \\
\text { nus canus, } \\
\text { Brachypterus } \\
\text { aurantiacus. }\end{array}$ & $\begin{array}{l}\text { Amérique } \\
\text { méridio- } \\
\text { nale, } \\
\text { Europe, } \\
\text { Asie. }\end{array}$ \\
\hline$?$ & 12 & $\begin{array}{l}\text { Les œufs sont isolément } \\
\text { disséminés directement } \\
\text { dans le parenchyme } \\
\text { interne. }\end{array}$ & $\begin{array}{l}\text { Celeus elegans, } \\
\text { Celeus flaves- } \\
\text { cens, Ceophlo- } \\
\text { eus lineatus, } \\
\text { Picus spec. }\end{array}$ & $\begin{array}{l}\text { Amérique } \\
\text { du Sud, } \\
\text { Amérique } \\
\text { méridio- } \\
\text { nale. }\end{array}$ \\
\hline 24 & $?$ & $\begin{array}{l}\text { Les œufs sont isolés dans } \\
\text { un parenchyme diffé- } \\
\text { rencié à petites cellules } \\
\text { avec gros noyaux. }\end{array}$ & $\begin{array}{l}\text { Colaptes aura- } \\
\text { tus, Meloner- } \\
\text { pes erythroce- } \\
\text { phalus. }\end{array}$ & $\begin{array}{l}\text { Amérique } \\
\text { du Nord, } \\
\text { Canada. }\end{array}$ \\
\hline $12-15$ & $19 \times 15$ & $\begin{array}{l}\text { Axe principal de la poche } \\
\text { du cirre parallèle au } \\
\text { bord latéral du seg- } \\
\text { ment. }\end{array}$ & $\begin{array}{l}\text { Lichtensteinipi- } \\
\text { cus funestris. }\end{array}$ & Asie. \\
\hline $10-12$ & $15 \times 12$ & $\begin{array}{l}\text { Les capsules utérines } \\
\text { sont isolément dissémi- } \\
\text { nées dans un paren- } \\
\text { chyme complètement } \\
\text { rempli de cellules d'as- } \\
\text { pect glandulaire. }\end{array}$ & $\begin{array}{l}\text { Gecinus nigri- } \\
\text { genis, Gecinus } \\
\text { chirrolophus. }\end{array}$ & Siam. \\
\hline
\end{tabular}

La poche du cirre, dont la musculature longitudinale est relativement puissante, mesure $96 \mu$ de long avec un diamètre maximum de $40 \mu$. Le canal déférent, fortement ondulé, s'étend, dorsalement, très loin et dépasse même légèrement le milieu du segment. Il est entouré de nombreuses glandes prostatiques. Assez étroit dans sa partie antiporale, le canal déférent se dilate rapidement pour atteindre bientôt un diamètre allant jusqu'à $24 \mu$. Pénétrant dans la poche du cirre, il semble y jouer le rôle de vésicule séminale interne, tandis que ses lacets externes celui de vésicule séminale externe.

Les testicules sont peu nombreux et volumineux. Nous en comptons environ 10 à 12. Sur les coupes transversales ils sont disposés en deux couches, mais sur les coupes horizontales en une rangée seulement, toute la moitié antérieure du parenchyme interne 
étant occupée par le grand développement de l'ovaire et du canal déférent. Latéralement on ne compte que deux testicules du côté antiporal. Légèrement comprimés par les glandes sexuelles femelles, les testicules ont généralement une forme ovale, mesurant $68 \mu$ $\times 32 \mu$.

L'ovaire, fortement lobé, prend rapidement des proportions considérables. Dans un segment de $924 \mu$ de large, il atteint une largeur de $420 \mu$. Dans les proglottis adultes il comprime postérieurement les testicules et parvient antérieurement au bord du segment. La glande vitellogère, médiane, est lobée et mesure $124 \mu \times 104 \mu$.

Le vagin passe en arrière de la poche du cirre et forme, presque au centre du segment, un réceptacle séminal fusiforme de $32 \mu \mathrm{d} t$

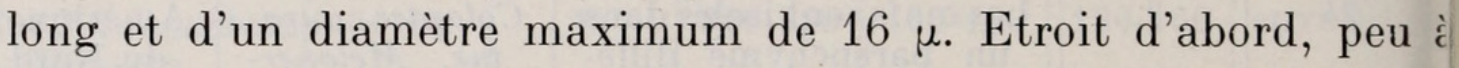
peu cependant le vagin se dilate jusqu'à un diamètre d'enviror $12 \mu$, puis, brusquement, en avant du réceptacle séminal, il st rétrécit considérablement.

Comme chez la plupart des Raillietina, il n'y a pas d'utéru: visible et les œufs semblent simplement disséminés dans le paren chyme. Dans les segments mûrs, chaque œuf est logé dans un capsule parenchymateuse. Ces capsules sont éparses, isolées ou parfois, en groupes de 2, 3 ou 4, dans un parenchyme différencié très dense, formé de grosses masses de cellules d'aspect glandulair qui se colorent très vivement comme des tissus glandulaires. Le capsules sont généralement presque sphériques, quoique asse souvent légèrement ovales. Les plus grandes mesurent $41 \mu \times 31 \mu$ L'oncosphère a $15,3 \mu \times 12 \mu$ et est entourée d'une membrane $d$ $25,5 \mu \times 17 \mu$.

D i s c u s s i o n: Les caractères spécifiques de cette nouvell espèce de Raillietina (Paroniella) des Piciformes sont: la longueu minime du strobila, comparée à celle des autres espèces du sous genre, la longueur des crochets qui atteint à peine la moitié de autres, le nombre très réduit des testicules et enfin la différen ciation typique du parenchyme dans les proglottis mûrs (voi le tableau comparatif de la page 172).

Si nous considérons le tableau comparatif des espèces de Raillit tina (Paroniella) des Piciformes, nous voyons qu'au premier abor notre espèce se rapproche sensiblement de $R$. (P.) cirroflex 
Tubangui et Masiluñgan, 1937, dont le strobila et les crochets sont également très courts et le nombre des testicules réduit comparativement aux autres espèces. Cependant, le nombre des testicules de $R$. (P.) cirroflexa est légèrement plus élevé, de même que celui de ses crochets qui, tout en étant relativement courts, sont plus longs que ceux de $R$. (P.) pinsonae. La différence entre ces deux espèces ressort encore mieux si l'on compare les ovaires. L'ovaire de $R$. (P.) cirrollexa est très petit ( $80 \mu$ de large), alors que celui de notre espèce est, au contraire, de taille considérable $(420 \mu)$ comme nous l'avons décrit. Nous retrouvons la même différence dans les dimensions des glandes vitellogènes. En outre, Tubangu I et Masiluñgan (1937) font remarquer la courbe particulière de la poche du cirre dont l'axe principal est parallèle au bord latéral du segment, sans que cette position inaccoutumée soit provoquée par la compression d'autres organes. Rien de tel n'a été remarqué chez notre espèce, malgré le grand développement de l'ovaire.

Pour les trois autres espèces connues, les différences sont trop manifestes pour qu'il soit nécessaire de les citer particulièrement.

\section{Biuterina fuhrmanni n. sp.}

Hôte: Emberiza aureola (Pall.) (Passeriformes).

Localité: Peking (Chine).

Comme le montre le tableau comparatif à la page 178, nous nous trouvons bien en présence d'une nouvelle espèce de Biuterina de Passeriformes.

La longueur totale de l'exemplaire est d'environ $35 \mathrm{~mm}$.

Le scolex est suivi d'une zône non segmentée d'environ 1,6 mm. de long et $140 \mu$ à $150 \mu$ de large. Les premiers segments, sans ébauches d'organes, sont plus larges que longs, environ $55 \mu$ à $70 \mu$ de long sur $145 \mu$ à $175 \mu$ de large. Il en est de même chez les proglottis jeunes qui varient entre $185 \mu$ à $200 \mu$ de long et $240 \mu$ à $250 \mu$ de large. Puis le rapport des dimensions est renversé et ce renversement s'accentue au fur et à mesure que l'on avance vers les segments mûrs. Les proglottis adultes mesurent $515 \mu$ à

Rev. Suisse de Zool., T. 48, 1941. 
$700 \mu$ de long et $350 \mu$ à $530 \mu$ de large; les segments gravides varient entre $700 \mu$ à $1100 \mu$ de long sur $500 \mu$ à $720 \mu$ de large.

Le scolex a un diamètre pouvant varier de $240 \mu$ à $320 \mu$. Il porte quatre ventouses d'un diamètre longitudinal de 124 à $130 \mu$ et d'un diamètre transversal de $100 \mu$ à $108 \mu$. Le rostre dont le diamètre est de $60 \mu$ est armé de 38 à 40 crochets, tous semblables, mesurant $19 \mu$ à $20 \mu$ de long sur $13 \mu$ à $14 \mu$ de base. Alors que sur deux scolex les crochets sont tombés, sur le troisième on voit nettement une couronne qui, fait remarquable, ne parait pas être double comme c'est le cas chez les autres espèces de Biuterina. Il semble, en effet, n'y avoir qu'une rangée de crochets, à moins que la distance entre les deux rangs soit si minime qu'elle n'ap. paraisse pas sur notre préparation. Néanmoins, c'est un caractère à retenir et qu'il serait intéressant de contrôler sur d'autres individus

Le système musculaire de même que le système nerveux n'onł rien de particulier.

$\mathrm{S}$ y s tè $\mathrm{me}$ e $\mathrm{x}$ c r éte u $\mathrm{r}$ : Les vaisseaux excréteurs ven traux sont assez gros, mesurent $36 \mu$ à $40 \mu$ de diamètre et son réunis, dans le bord postérieur de chaque proglottis, par un cana transversal.

Systèm e reproducteur: L'atrium génital est us grand et profond entonnoir, irrégulièrement alternant et débou chant dans le tiers antérieur du bord latéral du segment. La poch du cirre est légèrement oblique et dépasse le vaisseau excréteu Elle a la forme d'une poire mesurant $120 \mu$ à $120 \mu$ de long ave un diamètre maximum de $40 \mu$ à $44 \mu$. Dans les segments adulte le canal déférent forme une masse compacte de lacets au milie du proglottis, compris entre son bord antérieur et l'ovaire, puis s'allonge en ligne presque droite jusqu'à la poche du cirre. Dan les segments mûrs les lacets du canal déférent longent l'organ parutérin sur une certaine distance. Les testicules sont répart

Fig. 27-29, 31.

Biuterina fuhrmanni n. sp.: 27. crochets du rostre; 28. segment adult 29. segment avec organe parutérin jeune; 31. segment montrant l'orgal parutérin plus développé.

FIG. 30, 32-33.

Hymenolepis linderi n. sp.: 30. scolex; 32. coupe transversale d'un segme adulte; 33 . œuf. 
QUELQUES CESTODES NOUVEAUX D'OISEAUX D'ASIE
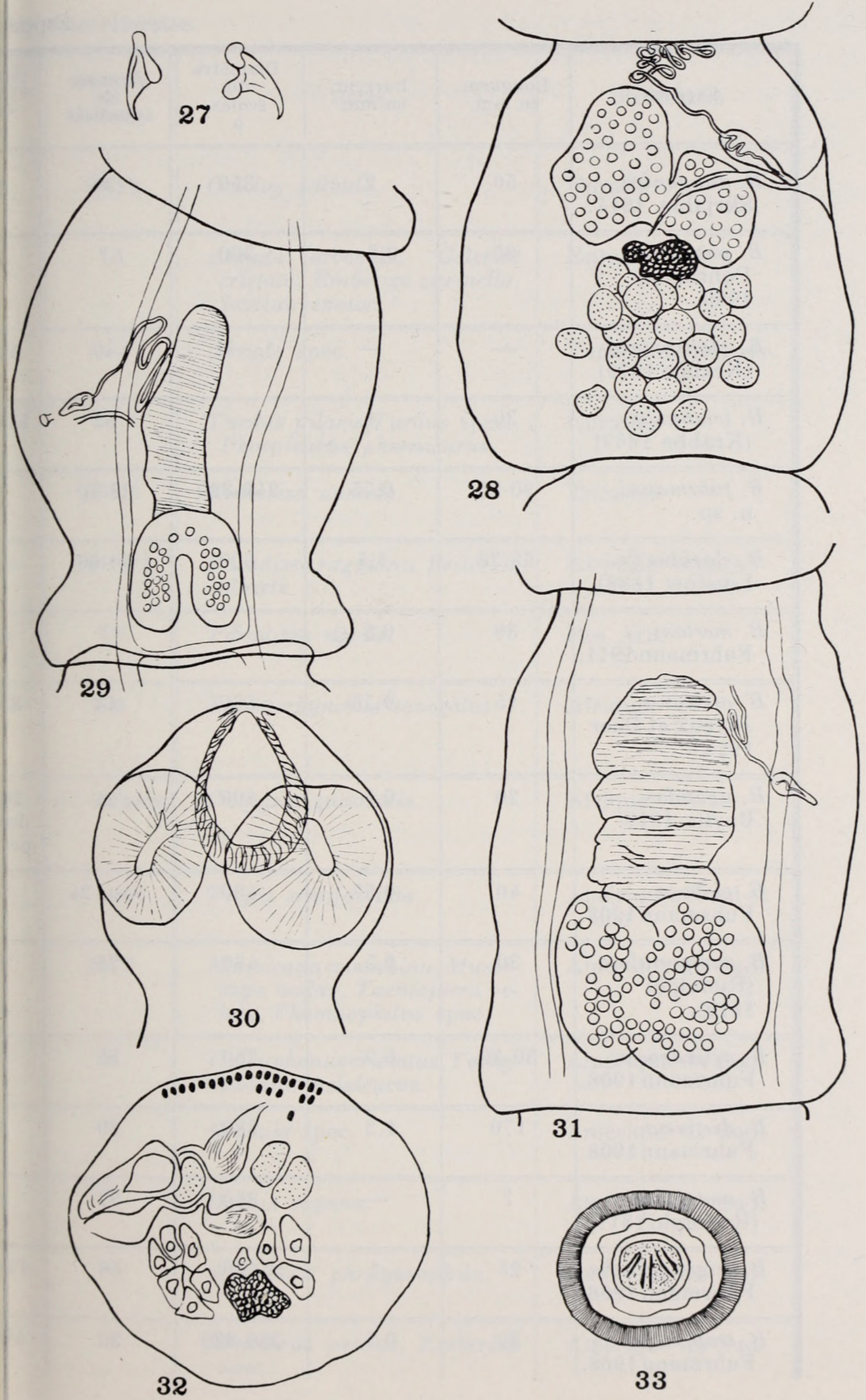
Espèce lu

\begin{tabular}{|c|c|c|c|c|c|}
\hline Espèces & $\begin{array}{l}\text { Longueur } \\
\text { en mm. }\end{array}$ & $\begin{array}{l}\text { Largeur } \\
\text { en } \mathrm{mm} \text {. }\end{array}$ & $\begin{array}{c}\text { Diamètre } \\
\text { du } \\
\text { scolex } \\
\mu\end{array}$ & $\begin{array}{l}\text { Nombre } \\
\text { de } \\
\text { crochets }\end{array}$ & $\begin{array}{c}\mathrm{L} \text { uect } \\
\mathrm{c} \text { hets }\end{array}$ \\
\hline $\begin{array}{l}\text { B. dunganica } \\
\text { Skrjabin } 1914 .\end{array}$ & 50 & 2 & 350 & 30 & 2 \\
\hline $\begin{array}{l}\text { B. passerina } \\
\text { Fuhrmann } \\
1908 .\end{array}$ & 80 & 0,6 & 300 & $?$ & 28 \\
\hline $\begin{array}{l}B \text {. planirostris } \\
\text { (Krabbe 1879). }\end{array}$ & - & 一 & - & 40 & 1 t 2. \\
\hline $\begin{array}{l}\text { B. triangula } \\
\text { (Krabbe 1869) }\end{array}$ & 30 & 1 & - & 32 & $55.38-$ \\
\hline $\begin{array}{l}\text { B. fuhrmanni } \\
\text { n. sp. }\end{array}$ & $30-35$ & 0,75 & $240-320$ & $38-40$ & -20 \\
\hline $\begin{array}{l}\text { B. clasulus (v. } \\
\text { Linstow 1888). }\end{array}$ & $60-70$ & 1,5 & 600 & $50-60$ & -12 \\
\hline $\begin{array}{l}\text { B. mertoni } \\
\text { Fuhrmann } 1911 .\end{array}$ & 30 & 0,5 & $?$ & $?$ & 9 \\
\hline $\begin{array}{l}B \text {. africana } \\
\text { Joyeux et Baer } \\
1928 \text {. }\end{array}$ & 15 & 0,75 & 500 & 44 & \{et 4 \\
\hline $\begin{array}{l}\text { B. ugandae } \\
\text { Baylis } 1919 .\end{array}$ & 20 & 0,7 & 400 & 44 & $\begin{array}{l}\text { ¿ et le } \\
\text { a res u } \\
\text { p moil }\end{array}$ \\
\hline $\begin{array}{l}\text { B. globosa } \\
\text { Fuhrmann } 1908 .\end{array}$ & 40 & 0,57 & 400 & env. 24 & $4-16$ \\
\hline $\begin{array}{l}\text { B. campanulata } \\
\text { (Rudolphi } \\
\text { 1819). }\end{array}$ & 30 & 0,5 & 480 & 26 & $\begin{array}{l}3 \cdot 46 \\
\text { et } \\
2-36 \\
\end{array}$ \\
\hline $\begin{array}{l}\text { B. cylindracea } \\
\text { Fuhrmann } 1908 .\end{array}$ & $30-40$ & 0,7 & 250 & 52 & $3-25$ \\
\hline $\begin{array}{l}\text { B. distincta } \\
\text { Fuhrmann } 1908 .\end{array}$ & 170 & 1,2 & 280 & 20 & 25 \\
\hline $\begin{array}{l}\text { B. motacillae } \\
\text { (Rudolphi 1819). }\end{array}$ & $?$ & - & 360 & 32 & 14 \\
\hline $\begin{array}{c}\text { B. trigonacantha } \\
\text { Fuhrmann } 1908 .\end{array}$ & 25 & 1 & 600 & 60 & $8-21$ \\
\hline $\begin{array}{l}\text { B. trapezoides } \\
\text { Fuhrmann } 1908 .\end{array}$ & 30 & 0,7 & $250-320$ & 30 & et 3 \\
\hline
\end{tabular}


les Passeriformes.

\begin{tabular}{|c|c|c|}
\hline $\begin{array}{l}\text { Nombre } \\
\text { de } \\
\text { testicules }\end{array}$ & Hôtes & Distribution \\
\hline $10-12$ & Oriolus galbula. & Eurasie. \\
\hline 10 & $\begin{array}{l}\text { Alauda arvensis, Galerita } \\
\text { cristata, Emberiza citrinella, } \\
\text { Lanius senator. }\end{array}$ & Eurasie. \\
\hline - & Alauda spec. & Turkestan, Eurasie. \\
\hline - & $\begin{array}{l}\text { Turdus pilaris, Turdus spec., } \\
\text { Phoenicurus phoenicurus. }\end{array}$ & Eurasie. \\
\hline $25-28$ & Emberiza aureola. & Eurasie. \\
\hline env. 32 & $\begin{array}{l}\text { Paradisea raggiana, Psilorhis } \\
\text { alberti. }\end{array}$ & Nouvelle-Guinée. \\
\hline 15 & Paradisea apoda. & Iles Aru. \\
\hline $5-8$ & Pomatorhynchus senegalus. & Afrique. \\
\hline 12 ou plus & Cinnyris gutturalis. & Afrique. \\
\hline - & Tityra semifasciata. & $\begin{array}{l}\text { Amériques Nord et } \\
\text { Sud. }\end{array}$ \\
\hline $8-10$ & $\begin{array}{l}\text { Muscicapacolumbina, Musci- } \\
\text { capa audax, Taenioptera ve- } \\
\text { lata, Thamnophilus spec. }\end{array}$ & Amérique du Sud. \\
\hline 8 & $\begin{array}{l}\text { Tachyphonus cristatus, Tachy- } \\
\text { phonus melaleucus. }\end{array}$ & Amérique du Sud. \\
\hline 12 & Gracula spec. & Amérique du Sud. \\
\hline$?$ & Dacnis cayana. & Amérique du Sud. \\
\hline 10 & Synallaxis phryganophila. & Amérique du Sud. \\
\hline$?$ & $\begin{array}{l}\text { Molothrus pecoris, Emberiza } \\
\text { spec. }\end{array}$ & Amérique du Sud. \\
\hline
\end{tabular}


sur deux couches compactes en arrière des glandes femelles. Ils occupent presque toute la moitié postérieure du proglottis adulte et atteignent le bord postérieur du segment. Leur nombre est de 25 à 28 et leur diamètre de $55 \mu$.

L'ovaire, volumineux, occupe une grande partie de la moitié antérieure du segment. Il est bilobé et le lobe antiporal est sensiblement plus grand que le lobe poral qui atteint la poche du cirre. La glande vitellogène, formée de trois lobes irréguliers se trouve en arrière de l'ovaire, dans l'isthme ovarien. Le vagin, cilié dans sa moitié distale, débouche dans l'atrium génital en arrière de la poche du cirre. Il a un diamètre d'environ $10 \mu$ à $12 \mu$.

L'utérus est bilobé. D'abord les lobes sont très distincts et largement séparés, donnant à l'utérus la forme caractéristique d'un fer à cheval. Au fur et à mesure que les œufs s'accumulent et mûrissent, les lobes de l'utérus s'élargissent en boules et la séparation disparaît presque totalement.

Dans les premiers segments où apparaît l'utérus, l'organe parutérin est allongé, digitiforme. Plus tard, au moment où les œuf vont passer de l'utérus dans l'organe parutérin, ce dernier s'élargit considérablement en même temps qu'il se godronne et forme de gros plis.

Dans les œufs mûrs, l'oncosphère a un diamètre de $28 \mu$ à $32 \mu$ L'embryon est entouré d'une première membrane interne mesurant $32 \mu$ à $36 \mu$, puis d'une membrane externe très fine, mesurant $40 \mu$ à $44 \mu$. Nous n'avons pas trouvé de proglottis dans lesquels les œufs avaient pénétré dans l'organe parutérin. Ce passage st produit sans doute seulement lorsque les proglottis sont détaché: du strobila, comme c'est le cas pour d'autres Biuterina.

D i s c u s s i o n : Notre espèce diffère de $B$. triangula (Krabbe par le nombre et surtout par la longueur des crochets, et par l fait qu'elle n'a qu'une couronne de crochets de taille égale. Ell diffère de $B$. planirostris (Krabbe) par la longueur et la forme de: crochets, de $B$. passerina Fuhrmann et $B$. dunganica Skrjabil essentiellement par le nombre des testicules et également par l: dimension des crochets. Mis à part le B. clasulus (v. Linstow) qu a environ 32 testicules, notre espèce diffère de tous les Biuterin connus jusqu'à ce jour, par le nombre élevé des testicules, soi 25 à 28 par segment. 
A propos de Biuterina motacillae brasiliensis Rudolphi 1819.

Rudolphi (1819) créa l'espèce Taenia motacillae brasiliensis. Cependant, nous remarquons que le genre d'Oiseaux Motacilla n'existe pas au Brésil. Si on compare la description et les mensurations de Rudolphi avec celles de Funrmann (1908) de Biuterina motacilla, on arrive à la conclusion que les deux espèces sont identiques. Il est done fort probable que le Cestode de Rudolphi ne provenait pas d'un Motacilla, mais d'un Oiseau de la même farnille, c'est-à-dire des Coerebidae. En conséquence, l'espèce de Rudolphi devra s'appeler $B$. motacillae (Rud. 1819) syn. $B$. motacilla Fuhrm. 1908.

\section{Hymenolepis linderi n. sp.}

Hôte: Syrrhaptes paradoxus (Pall.) (Pterocliformes).

Localité: Kuei Hua (Chine).

Dans ce groupe d'Oiseaux nous ne connaissons, à ce jour, que deux Cestodes du genre Hymenolepis: H. obvelata (Krabbe 1879) et $H$. rosenthali Mola 1913.

La longueur totale est d'environ $2 \mathrm{~mm}$., avec 110 à 150 proglottis.

Le scolex a un diamètre de $100 \mu$ à $120 \mu$. Il est armé de quatre ventouses d'un diamètre de $56 \mu$ à $60 \mu$ et d'un rostre de $48 \mu$ de long pour un diamètre maximum de $28 \mu$. La poche du rostre mesure $56 \mu$ de long et $40 \mu$ de diamètre. Ce rostre est caractérisé par le fait qu'il n'est pas armé de crochets, mais qu'il est complètement entouré de cellules glandulaires piriformes. Ces glandes secrètent probablement une substance facilitant la pénétration du scolex dans la muqueuse intestinale de l'hôte. Il est possible, aussi, que les crochets, chez les très nombreux exemplaires (plus de 100) soient tombés. Chez un individu long de $2 \mathrm{~mm}$., le strobila présente six régions: derrière le scolex nous avons une première région 
formée par le cou, zône non segmentée, de $120 \mu$ à $130 \mu$ de long sur environ $77 \mu$ de large, puis une deuxième, segmentée, avec des anneaux de $70 \mu$ à $80 \mu$ de large où nous trouvons les ébauches des organes génitaux, une troisième avec les segments jeunes mesurant $10 \mu$ à $16 \mu$ de long et $80 \mu$ à $120 \mu$ de large, une quatrième avec les segments adultes de $20 \mu$ de long sur $120 \mu$ à $150 \mu$ de large. La cinquième région est formée par les proglottis mûrissant longs de $20 \mu$ à $32 \mu$ et larges de $150 \mu$ à $200 \mu$. Finalement nous avons la région des segments gravides mesurant $48 \mu$ à $60 \mu$ de long sur $200 \mu$ à $300 \mu$ de large. Cependant, les 5 ou 6 derniers proglottis diminuent de largeur jusqu'à n'avoir que $120 \mu$ de large. Donc, tout au long de la segmentation, les proglottis sont sensiblement plus larges que longs. Dans la moitié postérieure de la région non segmentée on voit déjà apparaître les premières ébauches des organes sexuels. Les segments adultes sont peu nombreux; très tôt l'utérus fait son apparition et son développement ainsi que la régression des organes génitaux se font très rapidement. La poche du cirre subsiste assez longtemps encore, mais finalement elle aussi disparaît, laissant l'utérus envahir tout le parenchyme.

Les systèmes excréteurs et nerveux n'ont rien de particulier.

S y stèm e m u scula i re: Comme chez la plupart des Hymenolepis, la musculature est plutôt faible. Le système longitudinal est composé de deux couches de faisceaux comprenant peu de fibres chacun. La couche externe est formée de faisceaux nombreux et réguliers, tandis que la couche interne, chose exceptionnelle, est beaucoup plus pauvre. La musculature transversale et la musculature dorso-ventrale sont presque nulles.

Organes reproducteurs: L'atrium génital est unilatéral. La poche du cirre mesure $48 \mu$ à $56 \mu$ de long pour un diamètre maximum de $12 \mu$ à $14 \mu$. Sa paroi musculaire est très mince. Presque toute la moitié de la poche est occupée par une vésicule séminale interne. Peu avant d'aboutir à la poche du cirre, le canal déférent se dilate en une vésicule séminale externe très volumineuse qui peut être placée de deux manières, soit qu'elle se trouve allongée devant la poche du cirre, soit qu'elle est couchée, comme écrasée, contre la paroi dorsale de la poche (fig. 32).

La disposition des trois testicules est celle rencontrée généralement, c'est-à-dire un testicule poral au niveau de l'extrémité proximale de la poche du cirre et deux testicules antiporaux. Selon 
le degré de contraction du proglottis, ces testicules peuvent aussi être disposés soit en ligne droite, soit légèrement en triangle.

Le vagin, légèrement ondulé, passe ventralement par rapport à la poche du cirre. Au centre du segment, dans une légère dépression de l'ovaire, il se dilate en un énorme réceptacle séminal. Sur de nombreuses coupes on observe que le vagin s'élargit démesurément sur une certaine distance, immédiatement après l'atrium génital. Dans un proglottis adulte de $127 \mu$ de large, l'ovaire contenant quelques ovules géants mesure $55 \mu$ de large, et la glande vitellogène, médiane, a $22 \mu$ de large. L'utérus est sacciforme et dans les segments gravides il remplit tout le parenchyme. Le nombre d'œufs par proglottis mûr oscille entre 4 et 5 , jamais plus de 5 , généralement 4. Cependant, ce nombre peut descendre au-dessous de 4, car les premiers segments formés contiennent moins d'œufs que les segments suivants. Il en résulte que les 5 ou 6 derniers proglottis d'un individu complet sont moins larges et ne renferment que 3 ou même 2 œufs.

L'œuf a une structure spéciale et l'embryon est entouré de trois membranes: Une première très régulière, d'aspect lisse et brillante, une deuxième à paroi plissée fortement, et enfin une coque épaisse de $5 \mu$ à $8 \mu$, très foncée, donnant à l'œuf une forme assez régulièrement ovoïde. L'oncosphère a un diamètre de $22 \mu$ à $24 \mu$, la première membrane de $28 \mu$ à $30 \mu$, la deuxième de $32 \mu$ à $36 \mu$ et la troisième de $58 \mu$ à $60 \mu$. Dans l'oncosphère on voit les 6 crochets en trois groupes de 2 crochets très grands, mesurant $14 \mu$ à $15 \mu$ de long.

D is cussion: Alors que H. obvelata (Krabbe) et $H$. rosenthali Mola, possèdent chacun une couronne de 8 crochets, $H$. linderi n. sp. semble être inerme et son rostre non armé est, par contre, entouré de cellules glandulaires. Ceci constitue déjà un caractère particulier suffisant pour nous autoriser d'en faire une nouvelle espèce. En outre, alors que notre espèce a une longueur totale d'environ $2 \mathrm{~mm}$. pour une largeur maxima de 0,2 à $0,3 \mathrm{~mm}$., H. obvelata mesure $100 \mathrm{~mm}$. de long pour $0,5 \mathrm{~mm}$. de large et $H$. rosenthali $97 \mathrm{~mm}$. de long et $0,95 \mathrm{~mm}$. de large. Comme autres traits caractéristiques remarquables, nous avons le nombre très restreint des œufs et aussi leur structure particulière. Avec H. pauciovata, Fuhrmann avait également déjà décrit un type avec seule- 
ment 7 à 9 œufs géants. Un autre caractère rare dans le genre Hymenolepis est que toutes les coupes transversales de proglottis jeunes et adultes montrent des anneaux circulaires et non pas des anneaux aplatis dorso-ventralement comme c'est généralement le cas. Cependant, dès que l'utérus atteint un certain volume et que les œufs apparaissent en même temps que les organes génitaux régressent, les anneaux s'applatissent dorso-ventralement.

\section{Diorchis anomala n. sp.}

Hôte: Anas sp. (Anseriformes).

Localité: Wuchang (Chine).

Nos individus mesurent 60 à $70 \mathrm{~mm}$. de long. Cependant, s'ils sont complets, ils sont encore trop jeunes et, bien que l'utérus soit complètement développé, nous n'avons pas de segments gravides avec des œufs contenant une oncosphère. Nous en concluons que la longueur totale d'un individu n'est pas de 60 à $70 \mathrm{~mm}$., mais doit varier entre 80 et $100 \mathrm{~mm}$. Dans les 4 ou 5 derniers segments, c'est-à-dire dans les premiers segments formés par l'individu, l'utérus est presque vide, parfois le dernier segment est tout à fait stérile. C'est un phénomène assez fréquent chez certains Cestodes. Bien que tout au long du strobila, les segments soient plus larges que longs, au fur et à mesure qu'ils mûrissent ils s'allongent en diminuant de largeur.

Le scolex mesure $260 \mu$ de diamètre et $240 \mu$ de long. Il est pourvı de quatre ventouses ovalaires d'un diamètre longitudinal de $160 \mu$ et d'un diamètre transversal difficile à voir sur nos préparations mais qui semble ne devoir guère dépasser $100 \mu$. Le rostre, puissant a $184 \mu$ de long pour une largeur maxima de $100 \mu$. Il porte unt

FIG. 34-39.

Diorchis anomala n. sp.: 34. scolex; 35. segment adulte avec les organe mâles; 36 . crochets du rostre; 37 . segment adulte avec les organes femelles 38. coupe transversale d'un segment adulte; 39 . portion d'un strobil montrant l'atrophie de l'un des testicules. 
QUElQues Cestodes NOUVEAUX D'OISEAUX D'ASIE 185
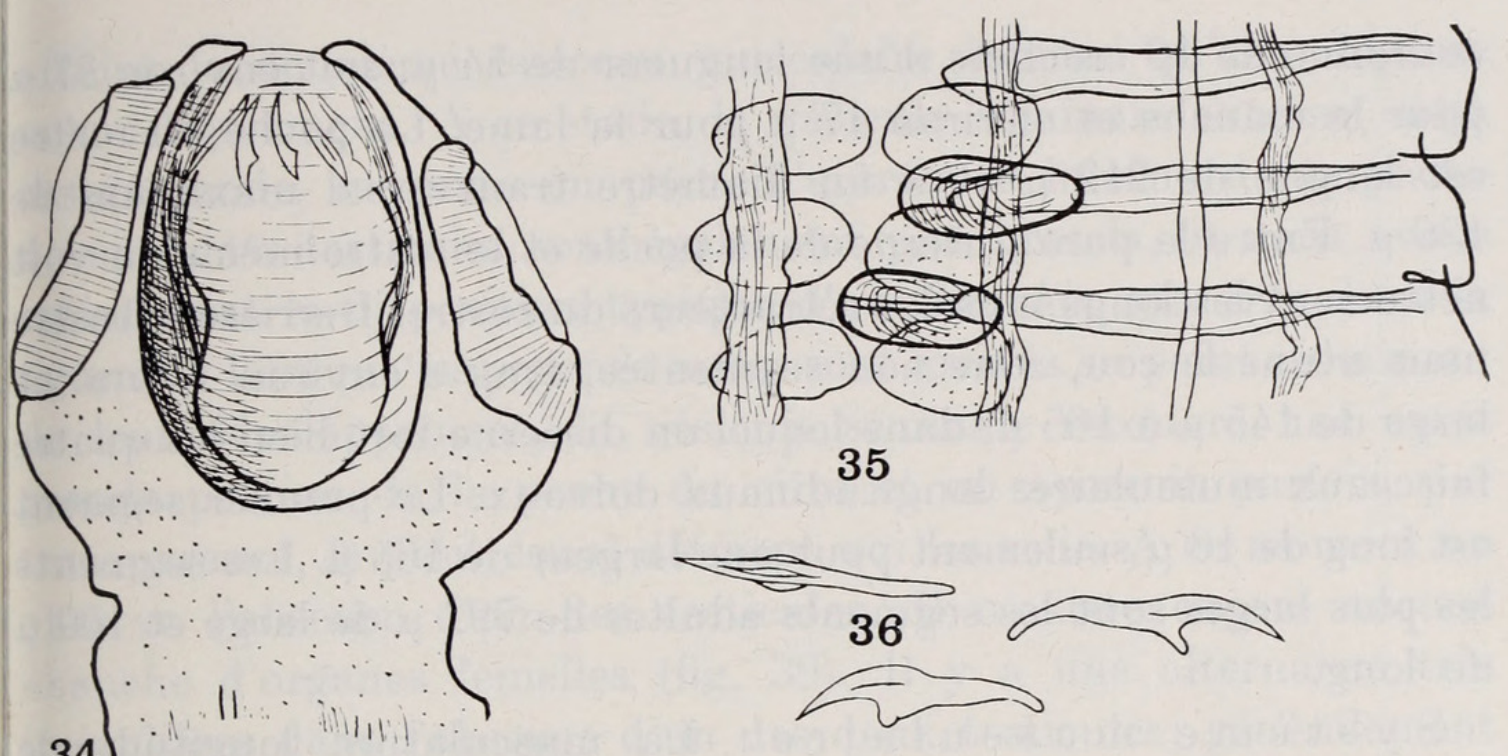

35
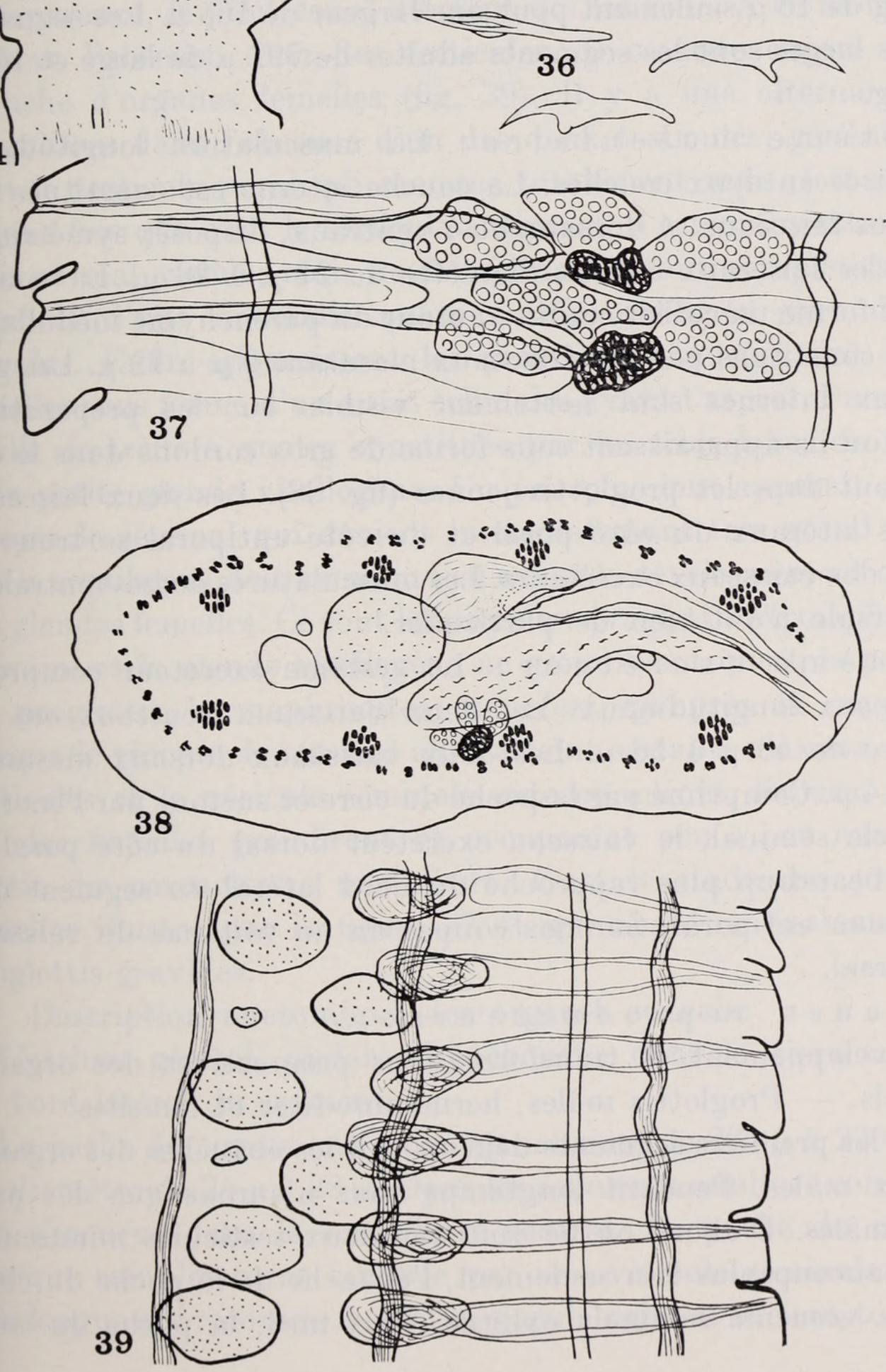
couronne de 10 crochets d'une longueur de $54 \mu$, soit environ $37 \mu$ pour le manche et environ $17 \mu$ pour la lame. La poche du rostre est longue de $212 \mu$ et a un diamètre transversal maximum de $136 \mu$. Entre la paroi interne de la poche et le rostre même on voit nettement les longs muscles rétracteurs du rostre. Derrière le rostre nous avons le cou, zône non segmentée, long d'environ $1 \mathrm{~mm}$. et large de $145 \mu$ à $165 \mu$, dans lequel on discerne fort bien les quatre faisceaux musculaires longitudinaux dorsaux. Le premier segment est long de $16 \mu$ seulement pour une largeur de $165 \mu$. Les segments les plus larges sont les segments adultes de $792 \mu$ de large et $100 \mu$ de long.

$\mathrm{S} \mathrm{y} \mathrm{stè} \mathrm{me} \mathrm{m} \mathrm{u} \mathrm{s} \mathrm{c} \mathrm{u} \mathrm{la} \mathrm{i} \mathrm{r} \mathrm{e} \mathrm{:} \mathrm{La} \mathrm{musculature} \mathrm{longitudinale}$ est divisée en deux couches. La couche interne est constituée par huit gros faisceaux, 4 dorsaux et 4 ventraux, disposés symétriquement. Ces faisceaux ont un diamètre de $24 \mu$ à $28 \mu$. La couche externe forme un collier continu autour du parenchyme médullaire; elle est constituée par des faisceaux mesurant $9 \mu$ à $12 \mu$. Les gros faisceaux internes sont nettement visibles sur les préparations totales où ils apparaissent sous forme de gros cordons dans le cou et surtout dans les proglottis jeunes (fig. 38). Les deux faisceaux les plus latéraux du côté poral et du côté antiporal se trouvent au-delà des vaisseaux excréteurs. Les musculatures dorso-ventrale et transversale n'ont rien de particulier.

$\mathrm{S}$ y s tè $\mathrm{m}$ e e x c r ét e u r : Le système excréteur comprend 4 vaisseaux longitudinaux. Les deux vaisseaux ventraux ont un diamiètre de $40 \mu$ à $56 \mu$. Les deux vaisseaux dorsaux mesurent $12 \mu$ à $14 \mu$. Comprimé par la poche du cirre et surtout par l'énorme réceptacle séminal, le vaisseau excréteur dorsal du côté poral se trouve beaucoup plus rapproché du bord latéral du segment que le vaisseau antiporal. Sur nos coupes on ne voit pas de vaisseau transversal.

Organes reproducteurs:

Développement et transformations progressives des organes sexuels. - Proglottis mâles, hermaphrodites et femelles:

Dans les premiers segments déjà on voit les ébauches des organes génitaux mâles. Pendant longtemps nous n'aurons que des proglottis mâles. D'abord on ne voit que deux testicules minuscules puis, beaucoup plus loin seulement, l'ébauche de la poche du cirre et de la vésucule séminale externe. Plus tard, la poche du cirre 
atteint le deuxième faisceau musculaire interne qu'elle dépasse même légèrement. A ce stade, la vésicule séminale externe, déjà assez grande, recouvre en partie l'extrémité interne de la poche du cirre; les testicules sont également plus grands, et nous avons maintenant la disposition typique d'un Diorchis, montrée dans la figure 35. Lorsque les organes mâles sont arrivés au point culminant de leur développement, la vésicule séminale recouvre une assez grande portion de la poche du cirre et on aperçoit parfois, par transparence, le petit canal déférent qui les relie. A ce moment on observe l'absence d'un des testicules. Il n'existe encore aucune ébauche d'organes femelles (fig. 39). Il y a une alternance très irrégulières dans l'absence d'un des deux testicules; généralement c'est le testicule poral qui manque totalement mais, fait curieux, chaque fois que c'est le testicule antiporal, il est rudimentaire, très petit et mal visible, mais ne fait jamais défaut complètement. Il s'agit là d'une anomalie qu'on a déjà observée chez d'autres Diorchis. Enfin apparaissent les premières ébauches des organes femelles sous forme d'un amas cellulaire ayant l'aspect d'une petite feuille de trèfle à quatre constituée par les trois lobes de l'ovaire et la petite glande vitellogène. Un peu plus loin apparaît aussi le réceptacle séminal qui devient immédiatement très grand du fait que la copulation doit avoir eu lieu avant le développement complet des glandes femelles. Ce sont les premiers proglottis hermaphrodites. L'ovaire et la glande vitellogène ne grandissent que très peu dans les proglottis hermaphrodites; ce n'est qu'après la copulation, lorsque le réceptacle séminal aura atteint sa taille maxima, que les testicules et la vésicule séminale externe auront disparu, que les glandes femelles grandiront et occuperont peu à peu une bonne partie du parenchyme médullaire. C'est le stade des proglottis femelles illustré par la figure 37 et qui aboutira finalement aux proglottis gravides.

Description anatomique des organes sexuels:

L'atrium génital, unilatéral, débouche dans le tiers antérieur du bord latéral du segment.

La poche du cirre a une longueur maxima de $200 \mu$ à $220 \mu$ pour un diamètre de $24 \mu$ à $28 \mu$. Dans les segments adultes (voir fig. 38) elle s'incurve dans le sens dorso-ventral. La poche du cirre est presque complètement occupée par une vésicule séminale interne très longue qui recouvre le ductus ejaculatorius et le cirre, tous deux 
Espèces du gen $D_{u \mid}$

\begin{tabular}{|c|c|c|c|c|}
\hline Espèces & $\begin{array}{l}\text { Longueur } \\
\text { en mm. }\end{array}$ & $\begin{array}{c}\text { Diamètre } \\
\text { du } \\
\text { scolex } \\
\mu\end{array}$ & $\begin{array}{l}\text { Nombre } \\
\text { de } \\
\text { crochets }\end{array}$ & $\begin{array}{r}\text { Long ur } \\
\text { d } \\
\text { croc s }\end{array}$ \\
\hline $\begin{array}{l}\text { D. flasescens } \\
\text { (Krefft 1871.) }\end{array}$ & $30-50$ & 195 & 10 & $6 \varepsilon$ \\
\hline $\begin{array}{l}\text { D. acuminata } \\
\text { Clerc } 1902 \text {. }\end{array}$ & env. 80 & $230-380$ & 10 & $27-$ \\
\hline $\begin{array}{l}\text { D. excentrica } \\
\text { Mayhew } 1925 .\end{array}$ & $26-52$ & env. 175 & 10 & 26 - \\
\hline $\begin{array}{l}\text { D. longicirrosa } \\
\text { Meggitt } 1927 .\end{array}$ & 14 & $200-240$ & 一 & - \\
\hline $\begin{array}{l}\text { D. bulbodes } \\
\text { Mayhew } 1929 .\end{array}$ & $60-70$ & 200 & 10 & 65 \\
\hline $\begin{array}{l}\text { D. kodonodes } \\
\text { Mayhew } 1929 .\end{array}$ & 156 & - & 一 & 1 \\
\hline $\begin{array}{l}\text { D. microcirrosa } \\
\text { Mayhew } 1929 .\end{array}$ & $25-33$ & 250 & 10 & $29 ?$ \\
\hline $\begin{array}{l}\text { D. spinata } \\
\text { Mayhew } 1929 .\end{array}$ & $80-122$ & env. 250 & 10 & 468 \\
\hline $\begin{array}{l}\text { D. spiralis } \\
\text { Szpotanska } 1931 .\end{array}$ & 20 & 一 & 一 & \\
\hline $\begin{array}{l}\text { D. formosensis } \\
\text { Sugimoto } 1934 .\end{array}$ & 一 & 一 & 一 & \\
\hline $\begin{array}{l}\text { D. nyrocae } \\
\text { Yamaguti } 1935 .\end{array}$ & $\begin{array}{c}14,2 \\
60\end{array}$ & $\begin{array}{c}150 \\
160-180\end{array}$ & 10 & $2: 7$ \\
\hline D. longae nom. nov. & $125-280$ & $289-348$ & 10 & \\
\hline D. anomala n. sp. & $80-100 ?$ & 260 & 10 & \\
\hline
\end{tabular}


ormes.

\begin{tabular}{|c|c|c|}
\hline $\begin{array}{l}\text { eur de la poche } \\
\text { du cirre } \\
\mu\end{array}$ & Hôtes & Distribution \\
\hline $270-350$ & $\begin{array}{l}\text { Anas superciliosa, Anas spec. } \\
\text { Aythya australis, Cairina } \\
\text { moschata, Dendrocygna ar- } \\
\text { cuata, Nettion castaneum, } \\
\text { Nettion crecca, Querquedula } \\
\text { gibberifrons, Spatula rhyn- } \\
\text { chotis, Casarca ferrugina. }\end{array}$ & Cosmopolite. \\
\hline $150-160$ & $\begin{array}{l}\text { Chaulelasmus streperus, Fuli- } \\
\text { gula affinis, Mareca pene- } \\
\text { lope, Nettion crecca. }\end{array}$ & Cosmopolite. \\
\hline $\begin{array}{l}\text { t le milieu du } \\
\text { ient. }\end{array}$ & Erismatura jamaicensis. & $\begin{array}{l}\text { Amérique du Nord et } \\
\text { Centrale, Antilles. }\end{array}$ \\
\hline $140-180$ & Nettion crecca. & Hémisphère Nord. \\
\hline $\begin{array}{l}\mathrm{t} \text { le milieu du } \\
\text { nent. }\end{array}$ & Oedemia fusca. & Région paléarctique. \\
\hline $\begin{array}{l}\text { tles trois quarts } \\
\text { segment. }\end{array}$ & Querquedula discors. & $\begin{array}{l}\text { Amérique du Nord, } \\
\text { Amérique Centrale, } \\
\text { Colombie, Equateur, } \\
\text { Pérou. }\end{array}$ \\
\hline $\begin{array}{l}\text { t le milieu du } \\
\text { nent. }\end{array}$ & Querquedula discors. & $\begin{array}{l}\text { Amérique du Nord, } \\
\text { Amérique Centrale, } \\
\text { Colombie,Equateur, } \\
\text { Pérou. }\end{array}$ \\
\hline $\begin{array}{l}\text { it entre le milieu } \\
\text { s trois quarts du } \\
\text { nent. }\end{array}$ & Chaulelasmus streperus. & Hémisphère Nord. \\
\hline 400 & Chenopis airata. & Australie. \\
\hline - & Anas platyrhyncha (dom.). & Asie. \\
\hline $\begin{array}{r}120-140 \\
60-100\end{array}$ & $\begin{array}{l}\text { Nyroca (Fuligula) marila } \\
\text { mariloides, Tadorna ta- } \\
\text { dorna. }\end{array}$ & Hémisphère Nord. \\
\hline 160 & Nyroca valisneria. & Amérique du Nord. \\
\hline $\begin{array}{l}200-220 \\
\text { eint pas le milieu } \\
\text { segment. }\end{array}$ & Anas sp. & Chine. \\
\hline
\end{tabular}


difficilement visibles. La vésicule séminale externe, très grande, mesure $100 \mu$ à $116 \mu$ de long et $64 \mu$ à $72 \mu$ de diamètre maximum. Sur une coupe horizontale passant par un segment hermaphrodite, elle occupe presque toute la longueur du segment. Les testicules sont ovalaires sur les sections horizontales avec un diamètre de $80 \mu$ à $88 \mu \times 45 \mu$ à $60 \mu$ et presque ronds sur les sections transversales où leur diamètre est de $80 \mu$ à $88 \mu \times 72 \mu$ à $80 \mu$.

Dans les segments femelles, l'ovaire atteint $220 \mu$ à $275 \mu \mathrm{de}$ large et $55 \mu$ à $65 \mu$ de long. Il est trilobé et les trois lobes se rejoignent au centre du proglottis. La glande vitellogène, médiane. mesure $60 \mu$ de large et $44 \mu$ de long.

Le vagin ne mesure guère plus de $60 \mu$ de long et débouche dans l'atrium génital en passant généralement du côté ventral de le poche du cirre. Brusquement il se dilate en un énorme réceptaclf séminal fusiforme, long de $180 \mu$ à $212 \mu$ pour un diamètre maximur de $44 \mu$ à $56 \mu$.

L'utérus apparait pour la première fois dans les segments adultes femelles sous forme d'un tube très étroit qui dépasse l'ovaire : gauche et à droite. Dans les segments mûrs, l'utérus sacciforme rempli presque tout le proglottis. Comme nous l'avons dit plus haut nos individus, tous trop jeunes encore, ne possèdent pas d'œuf: suffisamment mûrs pour permettre de faire une description et de: mensurations de l'œuf et de l'oncosphère.

D i s c us sion: Par sa longueur totale et le diamètre de sor scolex, Diorchis anomala n. sp. est identique à D. acuminat Clere, 1902, et à D. spinata Mayhew, 1929, mais il diffère des deu: espèces et par la longueur des crochets et par la longueur de li poche du cirre.

Quant aux neuf autres espèces, aucune confusion n'est possibl et il suffit, pour s'en rendre compte, de consulter le tableau compa ratif des espèces du genre Diorchis des Anseriformes à la page 188

A u sujet de Diorchis nyrocae Yamaguti 193 et Diorchis nyrocae Long et Wiggins 1939

En compulsant la littérature concernant les espèces du genr Diorchis des Anseriformes, nous avons trouvé deux espèces d 
même nom, soit Diorchis nyrocae Yamaguti 1935 et Diorchis myrocae Long et Wiggins 1939. Le nom proposé par Yamaguti ayant la priorité, nous nous sommes permis de changer le nom de l'espèce créée par Long et Wiggins en le dédiant à la première de ces deux auteurs, et nous l'avons appelé Diorchis longae nov. nom. pro Diorchis nyrocae Long et Wiggins, 1939, nec Yamaguti, 1935 (voir liste comparative à la page 188).

\section{Cladotaenia feuta Meggitt 1933.}

Hôtes: Circus assimilis (Jard.) Gypaetus barbatus (L.) $\}$ (Accipitriformes).

Localité: Indes-Chine.

En 1933 MegGitt créa le nom Cladotaenia feuta pour une espèce trouvée chez Circus assimilis (Jard.) en Inde. Il en a fait une description très sommaire; les crochets en particulier manquaient. D'après la figure qu'il a donnée d'un segment adulte, il apparaît que son individu était quelque peu contracté.

Dans notre collection de Chine nous avons trouvé, chez Gypaetus barbatus (L.) plusieurs Cestodes que, comparaison faite avec tous les Cladotaenia connus jusqu'à ce jour, nous avons identifié comme étant Cladotaenia feuta. Dans le tableau comparatif de la page 00 nous avons indiqué les mesures de MegGitt (1933) en même temps que les nôtres.

En étudiant tous nos individus, nous avons trouvé plusieurs scolex, dont un seul avec sa couronne de crochets complète, les autres ayant perdu tous leurs crochets. Le scolex avec crochets que nous avons reproduit dans la figure 41 est malheureusement un peu macéré et déformé. La plupart de nos échantillons étaient fortement contractés. Néanmoins, un individu particulièrement bien conservé nous a permis de donner les figures d'un segment adulte et d'un segment mûr plus complètes et surtout plus exactes dans la disposition et dans les proportions que le dessin de MEGGitT (1933).

Rev. Suisse de Zool., T. 48, 1941. 
A nat omie d u s colex: Nos scolex mesurent de $188 \mu$ à $220 \mu$ de large. Les ventouses, proéminantes, ont un diamètre de $70 \mu$ à $84 \mu$. Le diamètre du rostre, au niveau de la couronne des crochets, a $80 \mu$ à $100 \mu$ et est armé d'une double couronne de crochets au nombre très élevé de 92 à 96 . Les crochets du rang antérieur mesurent $25 \mu$ à $26 \mu$ de long et ceux du rang postérieur $18 \mu$ à $19 \mu$.

Organes reproducteurs: Alors que Meggrtt (1933) situe le pore génital dans le quart antérieur du proglottis, la figure 42 montre qu'il débouche à peu près au tiers antérieur du bord latéral du segment. Cette légère différence provient probablement de l'état de contraction de l'échantillon de Megaite (1933). L'atrium génital est irrégulièrement alternant.

La poche du cirre, en forme de poire, a $110 \mu$ à $145 \mu$ de long pour un diamètre de $45 \mu$ à $66 \mu$. Le canal déférent pénètre jusqu'au milieu du segment en formant une petite masse compacte de lacets dans le champ poral des testicules.

Les testicules au nombre de 84 à 86 sont disposés en deux longs champs (poral et antiporal) qui en contiennent chacun le même nombre et qui se réunissent en arrière de la glande vitellogène sur 3 à 4 rangées. Le diamètre des testicules varie de $33 \mu$ à $40 \mu$.

Le vagin passe en arrière de la poche du cirre; il est large de $10 \mu$. D'abord il chemine parallèlement à la poche du cirre et du canal déférent puis, un peu avant le milieu du proglottis, il descend brusquement en direction de l'utérus, formant presque un angle droit. Entre les deux lobes de l'ovaire, en arrière de l'utérus et en avant de la glande vitellogène, le vagin se dilate en un petit réceptacle séminal fusiforme.

L'ovaire a $80 \mu$ à $108 \mu$ de large et à peu près autant de long. La glande vitellogène, médiane, en arrière de l'ovaire, mesure $48 \mu$ à $60 \mu$ de large. L'utérus apparaît déjà tôt sous forme d'un petit tube s'allongeant tout droit entre les deux lobes ovariens. Dans les segments mûrs, l'utérus qui avance généralement très peu au-delà de la poche du cirre, forme de chaque côté 16 à 20 ramifi-

FIG. 40-43.

Cladotaenia feuta Meggitt: 40. crochets du rostre; 41. scolex; 42. segment adulte; 43. segment gravide. 
QUELQUES CESTODES NOUVEAUX D'OISEAUX D'ASIE
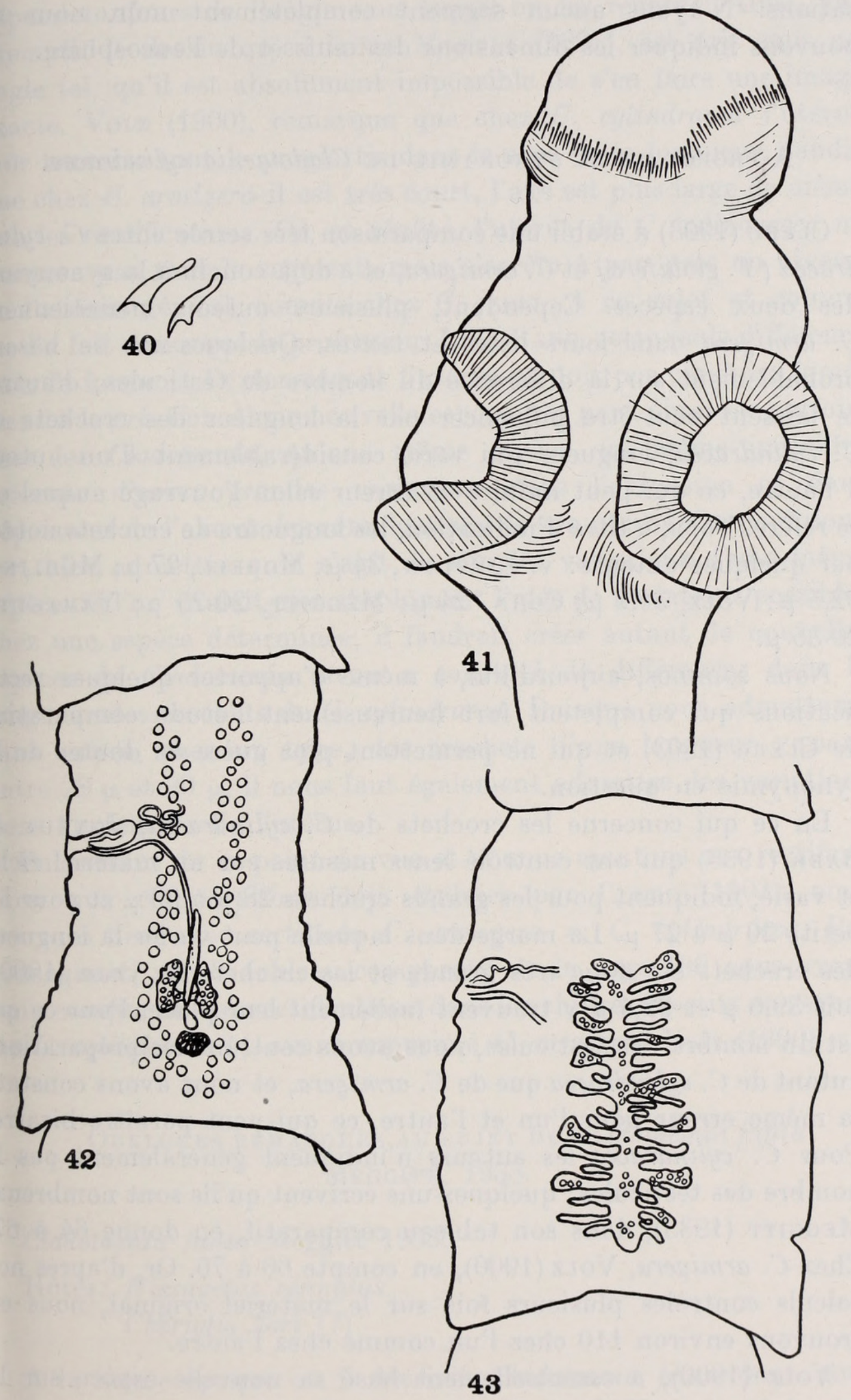
cations. N'ayant aucun segment complètement mûr, nous ne pouvons indiquer les dimensions des œufs et de l'oncosphère.

\section{A propos de la synonymie de Cladotaenia cylindracea.}

Clenc (1903) a établi une comparaison très serrée entre $C$. cylindracea ( $T$. globifera) et $C$. armigera, et a déjà conclu à la synonymie des deux espèces. Cependant, plusieurs auteurs maintiennent C. armigera dans leurs listes et textes. Quelques-uns se basent probablement sur la différence du nombre de testicules; d'autres se laissent peut-être influencer par la longueur des crochets de C. cylindracea, longueur qui varie considérablement d'un auteur à l'autre, ce qui peut induire en erreur selon l'ouvrage auquel on se réfère. Voici, à titre d'indication, les longueurs de crochets notées par quelques auteurs: v. Linstow, $34 \mu$; Morell, $27 \mu$; Mühling. $32,5 \mu$; Volz, 39,6 $\mu$; Cohn, $24 \mu$; Megaitt, 20-25 $\mu$; Yamaguti. $20-35 \mu$.

Nous sommes, aujourd'hui, à même d'apporter quelques rectifications qui complètent fort heureusement l'étude comparative de Clenc (1903) et qui ne permettent plus guère de douter de le synonymie en question.

En ce qui concerne les crochets de $C$. cylindracea, Joyeux ef BAEr (1936) qui ont contrôlé leurs mesures sur un matériel rich et varié, indiquent pour les grands crochets $28 \mu$ à $39 \mu$ et pour le: petits $20 \mu$ à $27 \mu$. La marge dans laquelle peut varier la longueu des crochets est donc très grande et les crochets de Vouz (1900) soit $39,6 \mu$ et $32,4 \mu$ y trouvent facilement leur place. Pour ce qu est du nombre des testicules, nous avons contrôlé des préparation autant de $C$. cylindracea que de $C$. armigera, et nous avons constat la même erreur pour l'un et l'autre, ce qui peut paraitre bizarre Pour $C$. cylindracea les auteurs n'indiquent généralement pas 1 nombre des testicules; quelques-uns écrivent qu'ils sont nombreux Megaitt (1933), dans son tableau comparatif, en donne 64 à 67 Chez C. armigera, Volz (1900), en compte 60 à 70. Or, d'après no calculs contrôlés plusieurs fois sur le matériel original, nous e trouvons environ $110 \mathrm{chez}$ l'un comme chez l'autre.

Volz (1900), a essentiellement basé sa nouvelle espèce sur l forme des crochets et de l'utérus mûr. Mais la forme des crochet 
dépend toujours de l'angle sous lequel on les regarde. Ainsi, par exemple, le dessin qu'en laisse Morell (1895), est pris sous un angle tel, qu'il est absolument impossible de s'en faire une image exacte. Volz (1900), remarque que chez $C$. cylindracea l'utérus mûr traverse tout le proglottis dans le sens de la longueur, tandis que chez $C$. armigera il est très court, l'axe est plus large de même que les ramifications. Or, en réalité, l'utérus de $C$. cylindracea ne traverse pas tout le segment, mais s'arrête à peu près au niveau de l'atrium génital, comme chez $C$. feuta. A ce sujet et comme me l'a fait remarqué le professeur J. G. BAER, cette seule différence dans la forme et l'extension de l'utérus ne doit pas conduire forcément à la création d'une nouvelle espèce. On peut observer parfois, chez les Cladotaenia, qu'une même espèce, un même individu, présente d'assez grandes variations dans l'extension et dans l'épaisseur de l'axe et des ramifications de son utérus. On peut donc fort bien admettre qu'il s'agit de simples variations d'une même espèce. Si l'on voulait nier absolument l'idée de variations possibles chez une espèce déterminée, il faudrait créer autant de nouvelles espèces de Cladotaenia qu'on a constaté de différences dans la longueur des crochets de $C$. cylindracea. Puisque nous admettons, sans autres, chez un type, des crochets d'une longueur variant entre $28 \mu$ et $39 \mu$, il nous faut également admettre des variations dans l'extension de l'utérus.

En partant de ce point de vue et si nous ajoutons nos rectifications aux comparaisons déjà établies par Clerc (1903), nous pouvons dire avec certitude $C$. armigera $=C$. cylindracea. Par conséquent, sur le tableau comparatif de la page 196 nous avons tenu compte de nos rectifications dans les. chiffres inscrits en regard de $C$. cylindracea et avons supprimé $C$. armigera Volz (1900).

\section{Quelques remarques au sujet de Cladotaemia fania MegGitt, 1933.}

Cladotaenia fania Meggitt 1933.

Hôtes: Hieraaetus rennatus.

Choriotis kori (?)

A la même époque où il décrivit Cladotaenia feuta dont nous avons complété l'étude ci-dessus, Megaitw (1933) créa également 
Espèces

\begin{tabular}{|c|c|c|c|c|}
\hline Espèces & $\begin{array}{l}\text { Longueur } \\
\text { de la poche } \\
\text { du cirre } \\
\mu\end{array}$ & $\begin{array}{l}\text { Diamètre } \\
\text { de la poche } \\
\text { du cirre } \\
\mu\end{array}$ & $\begin{array}{l}\text { Nombre de } \\
\text { crochets }\end{array}$ & 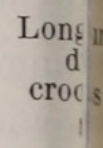 \\
\hline $\begin{array}{l}\text { C. cylindracea } \\
\text { (Bloch 1782). }\end{array}$ & $150-200$ & $59-69$ & 46 & $\begin{array}{r}28 \\
20\end{array}$ \\
\hline $\begin{array}{l}\text { C. feuta } \\
\text { Meggitt } 1933 .\end{array}$ & $\begin{array}{l}120-160 \\
110-145^{1}\end{array}$ & $\begin{array}{l}50-70 \\
45-66^{1}\end{array}$ & $\overline{92-96^{1}}$ & $\begin{array}{r}25 j \\
\text { et } 11\end{array}$ \\
\hline $\begin{array}{l}\text { C. fania } \\
\text { Meggitt } 1933 .\end{array}$ & $110-164$ & $72-100$ & 20 & $\epsilon$ \\
\hline $\begin{array}{l}\text { C. circi } \\
\quad \text { Yamaguti } 1935 .\end{array}$ & $120-150$ & 50 & 48 & 182 \\
\hline $\begin{array}{l}\text { C. accipitris } \\
\text { (Yamaguti 1935). } \\
\text { Syn. Paracladotaenia } \\
\text { accipitris. }\end{array}$ & 230 & 100 & - & \\
\hline $\begin{array}{l}\text { C. freani } \\
\text { Ortlepp } 1938 .\end{array}$ & 130 & 70 & $42-50$ & $\begin{array}{r}3.5 \\
\text { et }-2\end{array}$ \\
\hline $\begin{array}{l}\text { C. vulturi } \\
\text { Ortlepp } 1938 .\end{array}$ & $130-150$ & 80 & - & \\
\hline
\end{tabular}

1 Il s'agit des chiffres trouvés chez nos individus. Nous avons rescté Les légères différences s'expliquent facilement d'un individu à un autr

la nouvelle espèce Cladotaenia fania. Or, nous ne pouvons nou empêcher de remarquer que le nombre et la longueur des crochet qu'il indique sont tout à fait exceptionnels dans le genre Clado taenia et nous paraissent sujets à caution. Si nous faisons abstractiol des crochets, nous constatons que pour tout le reste de son anatomi $C$. fania est identique à $C$. cylindracea. Il serait donc intéressan de contrôler exactement le nombre et les dimensions des crochet et surtout de vérifier si le scolex et les crochets qui ont servi l'étude de Megarte (1933) appartiennent bien au strobila de so Cladotaenia et s'il n'y a pas eu, par hasard, confusion et mélang au moment de la mise en tube ou en faisant les préparation microscopiques. En effet, Megritt (1933) note lui-même que c'es 
des Accipitriformes.

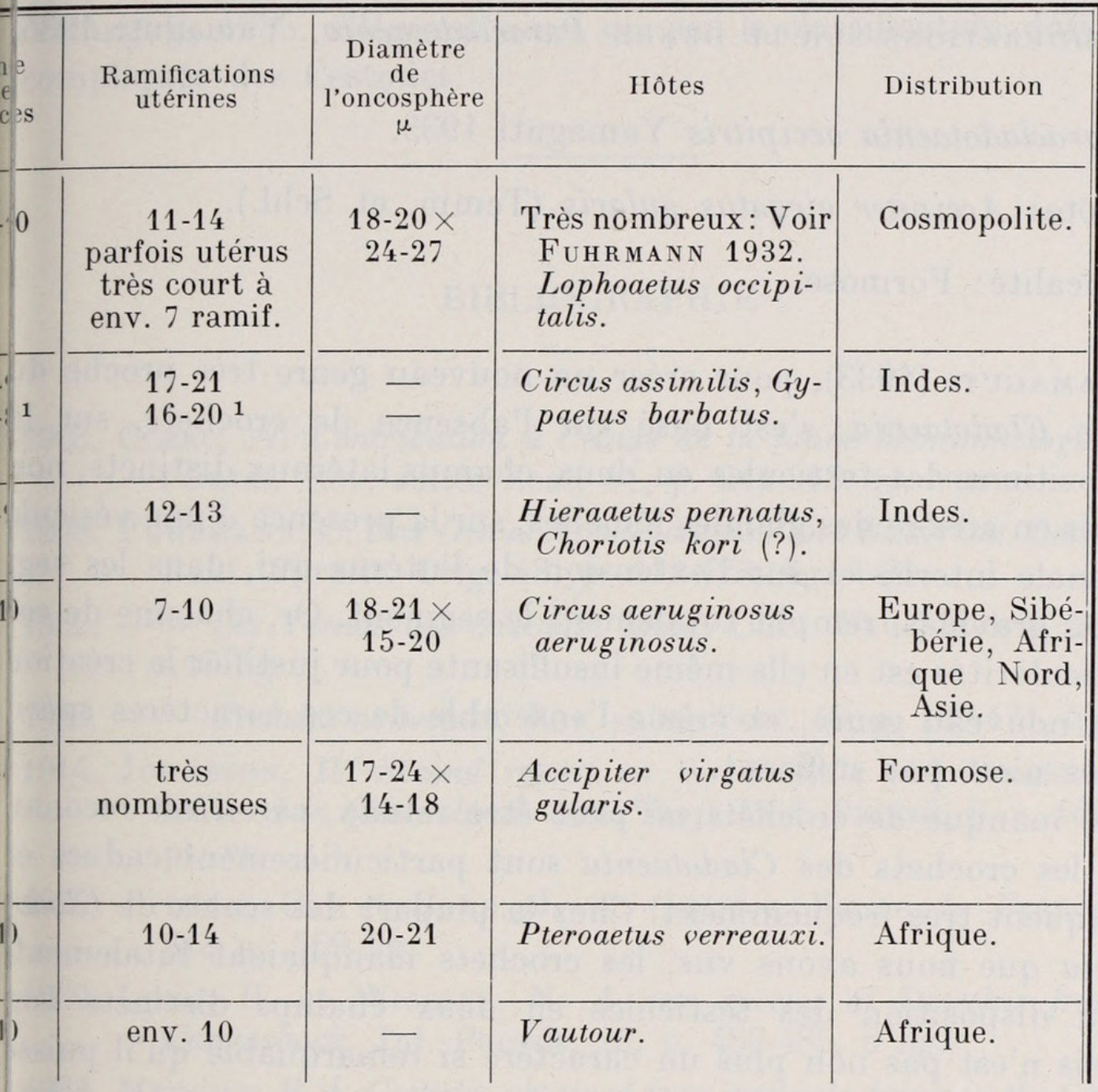

de Meggitt et avons seulement ajouté les nôtres à titre de comparaison.

a première fois qu'on rencontre un Cladotaenia chez un Charatriiforme. Ce seul fait nous fait déjà penser qu'il pourrait éventuelement y avoir eu confusion, car d'un côté il nous semble étonnant le trouver un Cladotaenia qui a l'anatomie de $C$. cylindracea chez Choriotis kori, et de l'autre côté le nombre et les dimensions des rochets de $C$. fania nous paraissent extraordinaires. Malheureusenent Megaitw (1933) ne donne pas le dessin et n'indique ni la orme ni le type de ces crochets.

Ne pouvant contrôler ces chiffres sur les matériaux originaux, hous nous contentons de faire remarquer ces particularités, mais naintenons momentanément $C$. fania dans le tableau comparatif i-dessus. 
Considérations sur Le genre Paracladotaenia, Yamaguti 1935.

Paracladotaenia accipitris Yamaguti 1935.

Hôte: Accipiter virgatus gularis (Temm. et Schl.).

Localité: Formose.

YAMAGUti (1933), pour créer un nouveau genre très proche d genre Cladotaenia, s'est basé sur l'absence de crochets, sur li dispositions des testicules en deux champs latéraux distincts, no1 réunis en arrière des glandes femelles, sur la présence d'une vésicul séminale interne et sur l'extension de l'utérus qui, dans les seg ments gravides, remplit totalement le segment. Or, chacune de ce particularités est en elle-même insuffisante pour justifier la créatio d'un nouveau genre, et même l'ensemble de ces caractères spéci fiques n'est pas suffisant.

Le manque de crochets ne peut être retenu, car il est reconn que les crochets des Cladotaenia sont particulièrement caducs $\epsilon$ manquent très fréquemment. Chez la plupart des scolex de Cladc taenia que nous avons vus, les crochets manquaient totalemen

La disposition des testicules en deux champs distincts no réunis n'est pas non plus un caractère si remarquable qu'il puiss permettre la création d'un genre, car on peut trouver la mêm disposition dans le genre Cladotaenia, notamment chez $C$. fani $C$. freani et $C$. vulturi.

La présence ou l'absence d'une vésicule séminale interne, pi plus que l'extension de l'utérus mûr, ne sauraient suffire por légitimer la création d'un genre nouveau. Par contre, ces quat: traits particuliers permettent de créer une nouvelle espèce c Cladotaenia fort intéressante. Ortlepp (1938) fait remarquer q si jamais un contrôle ultérieur prouvait que Paracladotaen accipitris était primitivement pourvu de crochets, $C$. freani $C$. vulturi devraient entrer dans le genre Paracladotaenia.

Plutôt que de transporter un jour $C$. freani et $C$. vulturi dans genre Paracladotaenia, nous trouvons préférable, plus logique plus pratique de supprimer ce nouveau genre en le faisant tomb en synonymie avec le genre Cladotaenia. C'est pourquoi nous avo ajouté, dans le tableau comparatif de la page 196, le Cladotaen: 
accipitris (Yamaguti 1935) Syn. Paracladotaenia accipitris Yamaguti, 1935, allégeant ainsi un peu la classification déjà trop compliquée des Cestodes.

\section{BIBLIOGRAPHIE}

1903. Cherc, W. Contribution à l'étude de la faune helminthologique de l'Oural. Rev. suisse Zool. 11, p. 241-368, pl. viII-XI.

1908. Funrmann, O. Das Genus Anonchotaenia und Biuterina. Centralbl. Bakt. Parasit. Orig. 48, p. 412-428, fig. 1-31.

1932. — Les Ténias des Oiseaux. Mém. Univ. Neuchâtel, 8, 381 p., 147 fig.

1933. - Cestodes nouveaux. Rev. suisse Zool. 40, p. 169-171, fig. 1-2. 1914. Johnston, H. Second report on the Cestoda and Acantocephala collected in Queensland. Ann. Trop. Med. Parasit. 8, p. 104-112, pl. vi.

1936. Joyeux, Ch. et Baer, J. G. Cestodes. Faune de France, 30, 613 p., 569 fig.

1939. Long, H. et Wiggins, N. A new species of Diorchis from the Canvasback. Jnl. Parasit. 25, p. 483-485, 6 fig.

1933. MegGitt, F. J. Cestodes obtained from animals dying in the Calcutta Zoological Gardens during 1931. Rec. Ind. Mus. 25, p. 145-165, fig. 1-21.

1895. Morell, A. Anatomische-Histologische Studien an Vogeltaenien. Arch. Naturg. Jahrg. 16, p. 81-102, 1 pl.

1938. Ortlepp, R. J. On two Cestodes recovered from a South-African Kite. Livr. Jub. Travassos, p. 353-358, 1 pl.

1819. Rudolphi, C. Entozoorum Synopsis. Berlin.

1937. Tubangui, M. et Masiluñgan, V. Tapexworm parasites of Philippine Birds. Phil. Journ. Sc. 62, p. 409-438, viI pl.

1900. Volz, W. Beitrag zur Kenntnis einiger Vogelcestoden. Arch. Naturg., p. 115-174, fig. 1-4, 4 pl.

1935. Yamaguti, S. Studies on the Helminth-Fauna of Japan; Part. 6 , Cestodes of Birds. I. Jap. Jnl. Zool. 6, p. 183-232, fig. 1-87. 


\section{$2 \mathrm{BHL}$ Biodiversity Heritage Library}

1941. "Quelques Cestodes nouveaux d'Oiseaux d'Asie." Revue suisse de zoologie 48, 143-199. https://doi.org/10.5962/bhl.part.117918.

View This Item Online: https://www.biodiversitylibrary.org/item/149511

DOI: https://doi.org/10.5962/bhl.part.117918

Permalink: https://www.biodiversitylibrary.org/partpdf/117918

\section{Holding Institution}

American Museum of Natural History Library

\section{Sponsored by}

BHL-SIL-FEDLINK

\section{Copyright \& Reuse}

Copyright Status: In copyright. Digitized with the permission of the rights holder.

Rights Holder: Muséum d'histoire naturelle - Ville de Genève

This document was created from content at the Biodiversity Heritage Library, the world's largest open access digital library for biodiversity literature and archives. Visit BHL at https://www.biodiversitylibrary.org. 